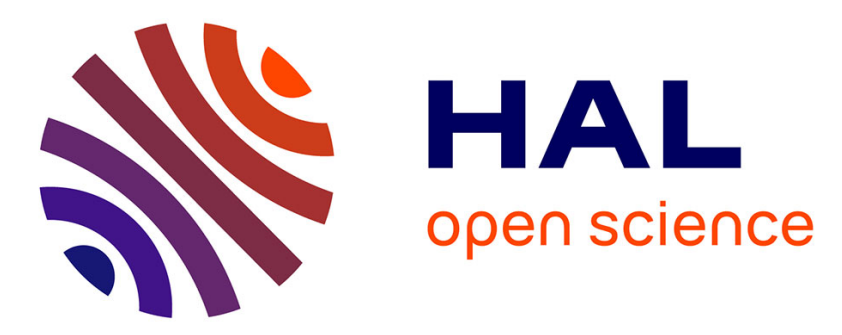

\title{
Extended Krein-Adler theorem for the translationally shape invariant potentials
}

David Gomez-Ullate, Yves Grandati, Robert Milson

\section{To cite this version:}

David Gomez-Ullate, Yves Grandati, Robert Milson. Extended Krein-Adler theorem for the translationally shape invariant potentials. Journal of Mathematical Physics, 2014, 55 (4), 10.1063/1.4871443 . hal-01513418

\section{HAL Id: hal-01513418 \\ https://hal.univ-lorraine.fr/hal-01513418}

Submitted on 9 May 2017

HAL is a multi-disciplinary open access archive for the deposit and dissemination of scientific research documents, whether they are published or not. The documents may come from teaching and research institutions in France or abroad, or from public or private research centers.
L'archive ouverte pluridisciplinaire HAL, est destinée au dépôt et à la diffusion de documents scientifiques de niveau recherche, publiés ou non, émanant des établissements d'enseignement et de recherche français ou étrangers, des laboratoires publics ou privés. 


\section{Extended Krein-Adler theorem for the translationally shape invariant potentials}

David Gomez-Ullate, Yves Grandati, and Robert Milson

Citation: Journal of Mathematical Physics 55, 043510 (2014); doi: 10.1063/1.4871443

View online: http://dx.doi.org/10.1063/1.4871443

View Table of Contents: http://aip.scitation.org/toc/jmp/55/4

Published by the American Institute of Physics

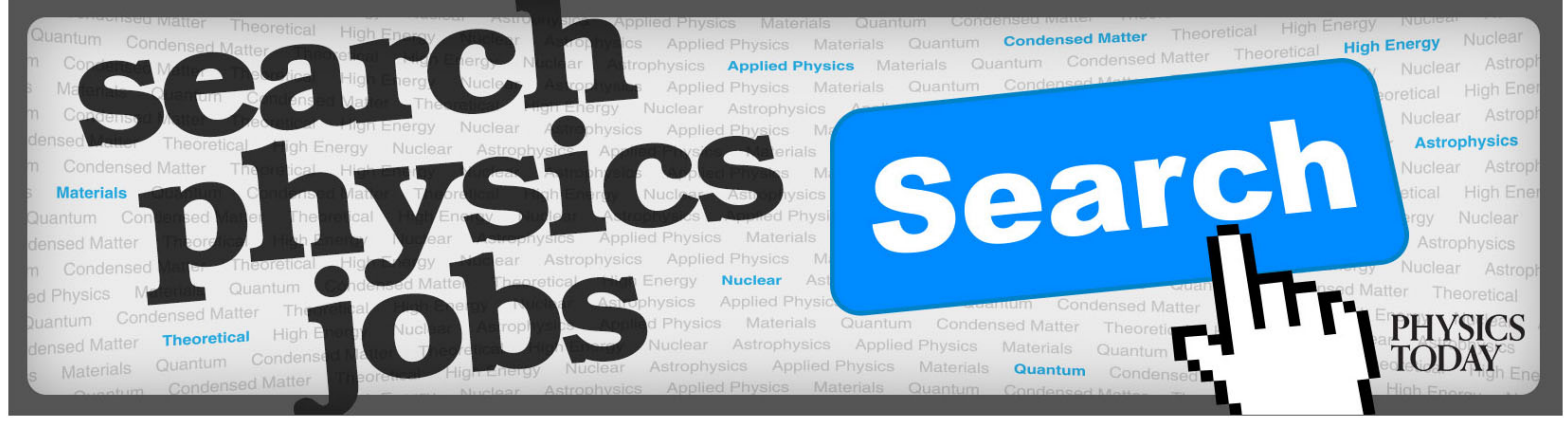




\title{
Extended Krein-Adler theorem for the translationally shape invariant potentials
}

\author{
David Gomez-Ullate, ${ }^{1}$ Yves Grandati, ${ }^{2}$ and Robert Milson ${ }^{3}$ \\ ${ }^{1}$ Departamento de Fisica Teorica II, Universidad Complutense de Madrid, \\ 28040 Madrid, Spain \\ ${ }^{2}$ LCP A2MC, Université de Lorraine, 1 Bd Arago, 57078 Metz, Cedex 3, France \\ ${ }^{3}$ Department of Mathematics and Statistics, Dalhousie University, Halifax, \\ Nova Scotia B3H 3J5, Canada
}

(Received 15 September 2013; accepted 3 April 2014; published online 28 April 2014)

Considering successive extensions of primary translationally shape invariant potentials, we enlarge the Krein-Adler theorem to mixed chains of state adding and state-deleting Darboux-Bäcklund transformations. It allows us to establish novel bilinear Wronskian and determinantal identities for classical orthogonal polynomials. (c) 2014 AIP Publishing LLC. [http://dx.doi.org/10.1063/1.4871443]

\section{INTRODUCTION}

In quantum mechanics, the construction of solvable potentials is a question of central interest and a subject of long time studies. The most efficient way to produce new systems which are exactly solvable in closed analytical form, starting from known ones is certainly the Darboux or DarbouxBäcklund transformation (DBT). ${ }^{1}$ Using one given eigenfunction of the initial potential (the seed function of the transformation), the DBT associates to this last a new potential, called an extension. It is then possible to enchain the DBT to generate chains of such extensions and in 1955, $\mathrm{Crum}^{2}$ has proven that for non-degenerate chains, both the extended potentials and their eigenfunctions can be written in terms of Wronskians of eigenfunctions of the initial potential (the seed functions of the chain). It has to be noticed that the factorization method in quantum mechanics, first introduced by Schrödinger ${ }^{3}$ and presented in its general form by Infeld and Hull, ${ }^{4}$ rests implicitly on a DBT scheme.

To go beyond the formal level and define equivalent boundary value problems for the systems related by a chain of DBT, it is necessary to ensure the regular character of the extended potentials. Crum $^{2}$ has shown that this is achieved if we use a complete chain, i.e., a set of seed functions which are consecutive eigenstates starting from the fundamental one. The obtained extensions correspond exactly to the hierarchy of superpartner potentials obtained in SUSY quantum mechanics. ${ }^{38,39} \mathrm{~A}$ more general answer has been provided shortly after by $\operatorname{Krein}^{57}$ and retrieved later by Adler. ${ }^{58}$ The so called Krein-Adler (KA) chains are also based on eigenfunctions of the initial potential but are lacunary with specific even gaps. The successive DBT in a KA chain lead to extensions in the spectrum of which some levels have been suppressed and for this reason they are said to be "state-deleting."

There is only a handful of classically known potentials for which it is possible to determine explicitly all the bound states in terms of elementary transcendental functions and to write explicitly their energies in terms of the quantum number. They all belong to category of translationally shape invariant potentials (TSIP) ${ }^{32,38-40}$ and they possess the characteristic feature to have bound states which are (up to a gauge factor) classical orthogonal polynomials in a suitable variable. Starting from these TSIP, the extensions generated by a KA chain are then rational in this variable.

During the four last years notable advances have been made in the study of rational extensions of TSIP and of their intimate connection with the recently discovered exceptional orthogonal polynomials (EOP). ${ }^{5-22,24-31,33-37,49-52}$ 
A systematic procedure to generate such extensions has been proposed. ${ }^{28-31,33-35}$ It rests on the use of DBT or chains of DBT based on unphysical seeds functions belonging to the disconjugacy sector of the initial equation, which are obtained from the eigenstates by a regularization procedure and which then share the quasi-polynomial character of the physical states. The regularization is achieved by the use of specific discrete symmetries of the original potential or, for finite bound state systems, by prolongation of the quantum number out of the physical domain of values. It allowed in particular to show the existence of infinite towers of strictly isospectral extensions which share with the original potential (then called a primary TSIP or PTSIP) the same shape invariance properties and constitute novel families of secondary TSIP. It has also led to the discovery of an enlarged shape invariance property satisfied by some families of extensions of potentials with finite bound state spectrum. ${ }^{33,51}$ The eigenstates of all these families of extended potentials are expressible in terms of various types of EOP. ${ }^{5-12}$

It appears that all the PTSIP possess at least one common discrete symmetry, called type 3 symmetry, which corresponds to a simultaneous change of the signs of all the parameters in which the potential depends. As opposed to the other symmetries (type 1 and 2) the DBT obtained by this symmetry generate only quasi-isospectral (or essentially isospectral) extensions since in their spectrum a supplementary level is added (state-adding DBT) for a negative integer value of the spectral parameter.

In this paper, we consider extensions of PTSIP built from mixed chains, i.e., containing stateadding and state-deleting DBT. The specific case of the harmonic oscillator, where the type 3 symmetry corresponds in fact to a "Wick rotation" of the independent variable, has been the subject of long time investigation. ${ }^{63,64,68,77,78}$ Oblomkov $^{72}$ has in particular shown that the extensions obtained from general mixed chains coincide with the rational extensions of the harmonic oscillator which possess the trivial monodromy property. He extended the seminal paper of Duistermaat and Grünbaum $^{73}$ and subsequent works ${ }^{74-76}$ to quadratically increasing rational potentials. In a recent work, we have proven that this set of extensions contains those which are associated to hamiltonians exactly solvable by polynomials and whose spectrum is subtended by systems of exceptional Hermite polynomials, ${ }^{42}$ providing a precise description of the properties of these EOP. ${ }^{79}$

We show here that every PTSIP possess a "reverse shape invariance property" which allows one to enlarge the Krein-Adler theorem to some mixed chains. This provides in particular new bilinear determinantal or Wronskians identities for the classical orthogonal polynomials. It has to be mentioned that during the making of the present article, parallel results although considered from a different point of view, have been presented in Ref. 23.

In the two first chapters, we recall basic facts concerning DBT and non-degenerate chains of DBT. Next, we give new explicit proofs of Wronskian formulas for the extended potentials and their eigenfunctions in the case of degenerate chains. In the following parts, we recall the regularity criterions for DBT and chains of DBT, in particular the Krein-Adler theorem. We then describe the essential features of TSIP and a way to use specific symmetries of PTSIP to generate their rational extensions. Using seed functions obtained via the type 3 symmetry (generators of state-adding DBT), we establish the reverse shape invariance condition associated to complete chains of such state-adding DBT. It allows us to prove an enlarged Krein-Adler theorem for mixed chains. Combining it with the general Wronskian formulas previously established, we give new bilinear Wronskian formulas for the eigenfunctions of the PTSIP. Finally, we apply these results to specific examples (harmonic and isotonic oscillators, trigonometric Darboux-Pöschl-Teller (TDPT) potential, Morse and KeplerCoulomb potentials) and show how to obtain new bilinear Wronskian or determinantal identities for Hermite, Laguerre, and Jacobi polynomials.

\section{FORMAL DBT AND CRUM FORMULAS}

We consider a one-dimensional Hamiltonian $\widehat{H}=-d^{2} / d x^{2}+V(x), x \in I \subset \mathbb{R}$ and the associated Schrödinger equation

$$
\psi_{\lambda}^{\prime \prime}(x)+\left(E_{\lambda}-V(x)\right) \psi_{\lambda}(x)=0
$$


$\psi_{\lambda}(x)$ being a formal eigenfunction of $\widehat{H}$ for the eigenvalue $E_{\lambda}$. In the following, we suppose that, with Dirichlet boundary conditions on $I, \widehat{H}$ admits a discrete spectrum of energies and eigenstates of the $\left(E_{n}, \psi_{n}\right)_{n \in\left\{0, \ldots, n_{\max }\right\} \in \mathbb{N}}$ where, without loss of generality, we can always suppose that the ground level of $\widehat{H}$ is zero $\left(E_{0}=0\right)$.

The Riccati-Schrödinger (RS) function $w_{\lambda}(x)=-\psi_{\lambda}^{\prime}(x) / \psi_{\lambda}(x)$ associated to $\psi_{\lambda}$ satisfies the corresponding RS equation ${ }^{32}$

$$
-w_{\lambda}^{\prime}(x)+w_{\lambda}^{2}(x)=V(x)-E_{\lambda} .
$$

If the set of general Riccati equations is invariant under the group $\mathcal{G}$ of smooth $S L(2, \mathbb{R})$-valued curves $\operatorname{Map}(\mathbb{R}, S L(2, \mathbb{R})),{ }^{53,54}$ the particular subclass of Riccati-Schrödinger equations is preserved by a specific subset of $\mathcal{G}$, called the DBT. Such transformation can be built from any solution $w_{\nu}(x)$

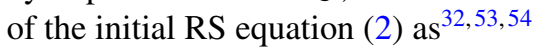

$$
w_{\lambda}(x) \stackrel{A\left(w_{v}\right)}{\rightarrow} w_{\lambda}^{(\nu)}(x)=-w_{v}(x)+\frac{E_{\lambda}-E_{v}}{w_{\nu}(x)-w_{\lambda}(x)},
$$

where $\lambda \neq v$.

$w_{\lambda}^{(\nu)}$ is then a solution of the RS equation

$$
-w_{\lambda}^{(v) \prime}(x)+\left(w_{\lambda}^{(v)}(x)\right)^{2}=V^{(v)}(x)-E_{\lambda},
$$

with the same energy $E_{\lambda}$ as in Eq. (2) but with a modified potential

$$
V^{(v)}(x)=V(x)+2 w_{v}^{\prime}(x),
$$

that we call an extension of $V(x)$.

To $w_{\lambda}^{(\nu)}$ corresponds an eigenfunction $\psi_{\lambda}^{(\nu)}=\exp \left(-\int d x w_{\lambda}^{(\nu)}(x)\right)$ of the extended hamiltonian $\widehat{H}^{(v)}=-d^{2} / d x^{2}+V^{(v)}(x)$, which can be written

$$
\psi_{\lambda}^{(v)}(x) \sim \widehat{A}\left(w_{\nu}\right) \psi_{\lambda}(x),
$$

where $\widehat{A}\left(w_{\nu}\right)$ is a first order operator given by

$$
\widehat{A}\left(w_{v}\right)=d / d x+w_{v}(x) .
$$

Equation (6) can then be rewritten as the Darboux-Crum formula

$$
\psi_{\lambda}^{(\nu)}(x) \sim \frac{W\left(\psi_{\nu}, \psi_{\lambda} \mid x\right)}{\psi_{\nu}(x)},
$$

where $W\left(y_{1}, \ldots, y_{m} \mid x\right)$ denotes the Wronskian of the family of functions $y_{1}, \ldots, y_{m}$

$$
W\left(y_{1}, \ldots, y_{m} \mid x\right)=\left|\begin{array}{ccc}
y_{1}(x) & \ldots & y_{m}(x) \\
\ldots & & \ldots \\
y_{1}^{(m-1)}(x) & \ldots & y_{m}^{(m-1)}(x)
\end{array}\right| .
$$

The eigenfunction $\psi_{\nu}$ is called the seed function of the DBT $A\left(w_{\nu}\right)$ and $V^{(v)}$ and $\psi_{\lambda}^{(\nu)}$ are the Darboux transforms of $V$ and $\psi_{\lambda}$, respectively.

Note that $A\left(w_{\nu}\right)$ annihilates $\psi_{\nu}$ and consequently the formulas (6) and (8) allow to obtain an eigenfunction of $V^{(v)}$ for the eigenvalue $E_{\lambda}$ only when $\lambda \neq v$. Nevertheless, we can readily verify that $1 / \psi_{v}(x)$ is such an eigenfunction. By extension, we then define the "image" by $A\left(w_{v}\right)$ of the seed eigenfunction $\psi_{v}$ itself as

$$
\psi_{v}^{(v)}(x) \sim \frac{1}{\psi_{v}(x)}
$$




\section{FORMAL CHAINS OF DBT}

At the formal level, the DBT can be straightforwardly iterated and a chain of $m$ DBT can be simply described by the following scheme:

$$
\left\{\begin{array}{l}
\psi_{\lambda} \stackrel{A\left(w_{v_{1}}\right)}{\longmapsto} \psi_{\lambda}^{\left(\nu_{1}\right)} \stackrel{A\left(w_{v_{2}}^{\left(N_{1}\right)}\right)}{\longmapsto} \psi_{\lambda}^{\left(N_{2}\right)} \ldots \stackrel{A\left(w_{v_{m}}^{\left(N_{m-1}\right)}\right)}{\longmapsto} \psi_{\lambda}^{\left(N_{m}\right)} \\
V \stackrel{A\left(w_{v_{1}}\right)}{\longmapsto} V^{\left(v_{1}\right)} \stackrel{A\left(w_{v_{2}}^{\left(N_{1}\right)}\right)}{\longmapsto} V^{\left(N_{2}\right)} \ldots \stackrel{A\left(w_{v_{m}-1}^{\left(N_{v_{m}}\right)}\right)}{\longmapsto} V^{\left(N_{m}\right)},
\end{array}\right.
$$

where $N_{j}$ denotes the $j$-uple $\left(v_{1}, \ldots, v_{j}\right)$ (with $\left.N_{1}=v_{1}\right)$ which completely characterizes the chain. We note $\left(N_{m}, v_{m+1}, \ldots, v_{m+k}\right)$ the chain obtained by adding to the chain $N_{m}$ the DBT associated to the successive eigenfunctions $\psi_{v_{m+1}}^{\left(N_{m}\right)}, \ldots, \psi_{v_{m+k}}^{\left(N_{m+k-1}\right)}$.

$\psi_{\lambda}^{\left(N_{m}\right)}$ is an eigenfunction associated to the eigenvalue $E_{\lambda}$ of the potential (see Eq. (5))

$$
V^{\left(N_{m}\right)}(x)=V(x)+2 \sum_{j=1}^{m}\left(w_{v_{j}}^{\left(N_{j-1}\right)}(x)\right)^{\prime}=V(x)-2 \sum_{j=1}^{m}\left(\log \left(\psi_{v_{j}}^{\left(N_{j-1}\right)}(x)\right)\right)^{\prime \prime}
$$

and can be written as (cf. Eqs. (6) and (8))

$$
\psi_{\lambda}^{\left(N_{m}\right)}(x)=\widehat{A}\left(w_{v_{m}}^{\left(N_{m-1}\right)}\right) \psi_{\lambda}^{\left(N_{m-1}\right)}(x)=\widehat{A}\left(w_{v_{m}}^{\left(N_{m-1}\right)}\right) \ldots \widehat{A}\left(w_{\nu_{1}}\right) \psi_{\lambda}(x),
$$

that is,

$$
\psi_{\lambda}^{\left(N_{m}\right)}(x)=\frac{W\left(\psi_{v_{m}}^{\left(N_{m-1}\right)}, \psi_{\lambda}^{\left(N_{m-1}\right)} \mid x\right)}{\psi_{\nu_{m}}^{\left(N_{m-1}\right)}(x)} .
$$

A chain is non-degenerate if all the spectral indices $v_{i}$ of the chain $N_{m}$ are distinct and is degenerate if some of them are repeated in the chain. For non-degenerate chains, $\mathrm{Crum}^{2}$ has derived very useful formulas for the extended potentials and their eigenfunctions in terms of Wronskians of eigenfunctions of the initial potential. ${ }^{59}$

\section{Crum's formulas:}

When all the $v_{j}$ and $\lambda$ are distinct, we have

$$
\psi_{\lambda}^{\left(N_{m}\right)}(x)=\frac{W^{\left(N_{m}, \lambda\right)}(x)}{W^{\left(N_{m}\right)}(x)}
$$

and

$$
V^{\left(N_{m}\right)}(x)=V(x)-2\left(\log W^{\left(N_{m}\right)}(x)\right)^{\prime \prime},
$$

where $W^{\left(N_{m}\right)}(x)=W\left(\psi_{v_{1}}, \ldots, \psi_{\nu_{m}} \mid x\right)$.

A very direct proof of this result can be obtained by using the Jacobi identity for Wronskians Jacobi formula. ${ }^{56}$

Let $A=\left[a_{i j}\right]$ be a $n \times$ nmatrix. We note $A_{1 \ldots n}^{1 \ldots n}$ the determinant of $A$ and $A_{q_{1} \ldots q_{r}}^{p_{1} \ldots p_{r}}$ the retainer minor of order $r$ which is the minor obtained from $A$ by deleting the lines $\{1, \ldots, n\} /\left\{p_{1} \ldots p_{r}\right\}$ and to the columns $\{1, \ldots, n\} /\left\{q_{1} \ldots q_{r}\right\}$. The Jacobi identity reads

$$
A_{1 \ldots n}^{1 \ldots n} A_{1 \ldots n-2}^{1 \ldots n-2}-A_{1 \ldots n-1}^{1 \ldots n-1} A_{1 \ldots n-2, n}^{1 \ldots n-2, n}+A_{1 \ldots n-2, n}^{1 \ldots n-2, n-1} A_{1 \ldots n-2, n-1}^{1 \ldots n n-n}=0 .
$$

This leads to the following Wronskian Jacobi formula:

$$
W\left(y_{1}, \ldots, y_{n} \mid x\right)=\frac{W\left(W\left(y_{1}, \ldots, y_{n-1}\right), W\left(y_{1}, \ldots, y_{n-2}, y_{n}\right) \mid x\right)}{W\left(y_{1}, \ldots, y_{n-2} \mid x\right)} .
$$


Proof. It is a direct consequence of the Jacobi identity if we take $A_{1 \ldots n}^{1 \ldots n}=W\left(y_{1}, \ldots, y_{n} \mid x\right)$. Then

$$
A_{1 \ldots n-2, n}^{1 \ldots n-2, n}=\left|\begin{array}{cccc}
y_{1} & \ldots & y_{n-2} & y_{n} \\
\ldots & & \ldots & \ldots \\
y_{1}^{(n-2)} & \ldots & y_{n-2}^{(n-2)} & y_{n}^{(n-2)} \\
y_{1}^{(n)} & \ldots & y_{n-2}^{(n)} & y_{n}^{(n)}
\end{array}\right|=W^{\prime}\left(y_{1}, \ldots, y_{n-2}, y_{n} \mid x\right)
$$

and in the same manner

$$
A_{1 \ldots n-2, n}^{1 \ldots n-2, n-1}=W^{\prime}\left(y_{1}, \ldots, y_{n-1}\right)
$$

Proof of the Crum formulas: Using in the Wronskian Jacobi identity the following property of Wronskians: ${ }^{55}$

$$
W\left(u y_{1}, \ldots, u y_{m} \mid x\right)=u^{m} W\left(y_{1}, \ldots, y_{m} \mid x\right)
$$

this gives

$$
\frac{W\left(y_{1}, \ldots, y_{m}, y \mid x\right)}{W\left(y_{1}, \ldots, y_{m} \mid x\right)}=\frac{W\left(\frac{W\left(y_{1}, \ldots, y_{m}\right)}{W\left(y_{1}, \ldots, y_{m-1}\right)}, \frac{W\left(y_{1}, \ldots, y_{m-1}, y\right)}{W\left(y_{1}, \ldots, y_{m-1}\right)} \mid x\right)}{\frac{W\left(y_{1}, \ldots, y_{m} \mid x\right)}{W\left(y_{1}, \ldots, y_{m-1} \mid x\right)}}
$$

and comparing to Eq. (14), we obtain the Crum formula for the eigenfunctions Eq. (15). Inserting this result in Eq. (12), we then deduce the Crum formula for the potential Eq. (16).

The eigenfunctions $\psi_{v_{1}}, \ldots, \psi_{v_{m}}$ of $V$ are called the seed functions of the chain of DBT associated to the $m$-uple of spectral indices $N_{m}=\left(v_{1}, \ldots, v_{m}\right)$.

\section{WRONSKIAN FORMULAS FOR THE IMAGES OF THE SEED FUNCTIONS}

Being obtained by a recursion which starts from the one step Wronskian formula (8), this last being not relevant when $\lambda=v$, the Crum formulas are restricted to the values of $\lambda \notin N_{m}$. Nevertheless, we can also obtain Wronskian formulas for the image by a chain of DBT of one of the seed functions associated to the chain. First note that, to obtain $\psi_{v_{k}}^{\left(N_{m}\right)}, 1 \leq k<m$, we can make use the Crum formulas if we start only from the $(k+1)$ th step. By using the eigenfunctions $\left(\psi_{v_{k+1}}^{\left(N_{k}\right)}, \ldots, \psi_{v_{m}}^{\left(N_{k}\right)}\right)$ of $V^{\left(N_{k}\right)}, N_{k}=\left(v_{1}, \ldots, v_{k}\right)$, as seeds functions and apply the corresponding chain of $m-k$ DBT to (see Eq. (10))

$$
\psi_{v_{k}}^{\left(N_{k}\right)} \sim \frac{1}{\psi_{v_{k}}^{\left(N_{k-1}\right)}}=\frac{W^{\left(N_{k-1}\right)}(x)}{W^{\left(N_{k}\right)}(x)}
$$

we obtain

$$
\psi_{v_{k}}^{\left(N_{m}\right)}(x)=\frac{W\left(\psi_{v_{k+1}}^{\left(N_{k}\right)}, \ldots, \psi_{v_{m}}^{\left(N_{k}\right)}, \psi_{v_{k}}^{\left(N_{k}\right)} \mid x\right)}{W\left(\psi_{v_{k+1}}^{\left(N_{k}\right)}, \ldots, \psi_{v_{m}}^{\left(N_{k}\right)} \mid x\right)}
$$

Lemma 1. Consider the formal non-degenerate chain of DBT characterized by the m-uple of spectral indices $N_{m}=\left(v_{1}, \ldots, v_{m}\right)$. If $\left\{v_{j_{1}}, \ldots, v_{j_{l}}\right\}$ is a subset of $\left\{v_{1}, \ldots, v_{m}\right\}, N_{m} / v_{j_{1}}, \ldots, v_{j_{l}}$ means the $(m-l)$-uple obtained by suppressing into $\left(v_{1}, \ldots, v_{m}\right)$ the elements $\left(v_{j_{1}}, \ldots, v_{j_{l}}\right)$. Then, for any $1 \leq k \leq$ mwe have

$$
\psi_{v_{k}}^{\left(N_{m}\right)}(x) \sim \frac{W^{\left(N_{m} / v_{k}\right)}(x)}{W^{\left(N_{m}\right)}(x)},
$$

the ordering of the successive indices in $N_{m}$ being unimportant. 
Proof: In the particular case $k=m$, taking account of Eqs. (10) and (15), the result is immediate

$$
\psi_{v_{m}}^{\left(N_{m}\right)}(x) \sim \frac{1}{\psi_{v_{m}}^{\left(N_{m-1}\right)}(x)} \sim \frac{W^{\left(N_{m} / v_{m}\right)}(x)}{W^{\left(N_{m}\right)}(x)} .
$$

It results that if the ordering of the successive DBTs in the chain is, as in the case of the usual Crum formulas, unimportant (up to a constant factor), Eq. (26) implies Eq. (25). We now proceed by induction to show that is indeed the case.

For $m=2, N_{2}=\left(v_{1}, v_{2}\right)$, using standard properties of the Wronskians, ${ }^{55}$ Eqs. (8) and (10), we deduce

$$
\psi_{\nu_{1}}^{\left(N_{2}\right)}(x)=\frac{W\left(\psi_{\nu_{1}}^{\left(\nu_{1}\right)}, \psi_{\nu_{2}}^{\left(\nu_{1}\right)} \mid x\right)}{\psi_{\nu_{2}}^{\left(\nu_{1}\right)}(x)} \sim \frac{W\left(1, W\left(\psi_{\nu_{1}}, \psi_{\nu_{2}}\right) \mid x\right)}{\psi_{\nu_{1}}(x) W\left(\psi_{\nu_{1}}, \psi_{\nu_{2}} \mid x\right)} \sim \frac{W^{\prime}\left(\psi_{\nu_{1}}, \psi_{\nu_{2}} \mid x\right)}{\psi_{\nu_{1}}(x) W\left(\psi_{v_{1}}, \psi_{\nu_{2}} \mid x\right)} .
$$

With the help of the Wronskian theorem ${ }^{80}$

$$
W^{\prime}\left(\psi_{v_{1}}, \psi_{\nu_{2}} \mid x\right)=\left(E_{v_{1}}-E_{v_{2}}\right) \psi_{v_{1}}(x) \psi_{\nu_{2}}(x)
$$

Eq. (27) can be rewritten

$$
\psi_{v_{1}}^{\left(N_{2}\right)}(x) \sim \frac{\psi_{\nu_{2}}(x)}{W\left(\psi_{v_{1}}, \psi_{v_{2}} \mid x\right)} \sim 1 / \psi_{\nu_{1}}^{\left(v_{2}\right)}(x)=\psi_{v_{1}}^{\left(v_{2}, \nu_{1}\right)}(x) .
$$

Suppose then Eq. (25) is true for a $m$-step DBT. Consider then a $(m+1)$-step chain. Since at the preceding step, the ordering is not important, we can write

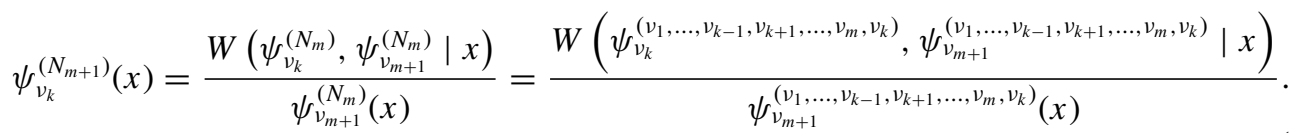

This can be rewritten

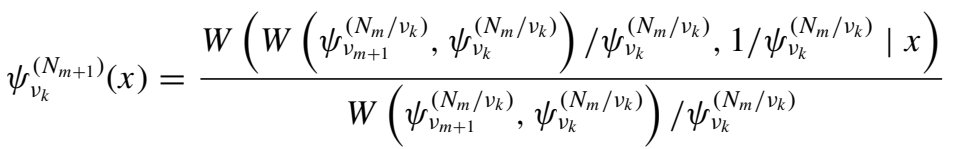

$$
\begin{aligned}
& =\frac{W\left(1, W\left(\psi_{v_{m+1}}^{\left(N_{m} / \nu_{k}\right)}, \psi_{\nu_{k}}^{\left(N_{m} / v_{k}\right)}\right) \mid x\right)}{\psi_{v_{k}}^{\left(N_{m} / v_{k}\right)}(x) W\left(\psi_{v_{m+1}}^{\left(N_{m} / v_{k}\right)}, \psi_{v_{k}}^{\left(N_{m} / v_{k}\right)} \mid x\right)} \\
& =-\frac{W^{\prime}\left(\psi_{v_{m+1}}^{\left(N_{m} / v_{k}\right)}, \psi_{\nu_{k}}^{\left(N_{m} / v_{k}\right)} \mid x\right)}{\psi_{v_{k}}^{\left(N_{m} / v_{k}\right)}(x) W\left(\psi_{v_{m+1}}^{\left(N_{m} / \nu_{k}\right)}, \psi_{\nu_{k}}^{\left(N_{m} / v_{k}\right)} \mid x\right)},
\end{aligned}
$$

that is, using the Wronskian theorem ${ }^{80}$

$$
\psi_{v_{k}}^{\left(N_{m+1}\right)}(x) \sim \frac{\psi_{v_{m}+1}^{\left(N_{m} / v_{k}\right)}(x)}{W\left(\psi_{v_{k}}^{\left(N_{m} / \nu_{k}\right)}, \psi_{v_{m+1}}^{\left(N_{m} / \nu_{k}\right)} \mid x\right)} \sim \frac{1}{\psi_{v_{k}}^{\left(N_{m} / v_{k}\right)}}
$$

The property $(25)$ is also verified for $(m+1)$-step DBT, which ensures that it is verified for any number of steps.

\section{DEGENERATE CHAINS}

Consider first the case of a two-step DBT with two instances of the same seed function. We have from Eq. (10)

$$
V^{\left(v_{1}, v_{1}\right)}(x)=V^{\left(\nu_{1}\right)}(x)-2\left(\log \psi_{\nu_{1}}^{\left(\nu_{1}\right)}(x)\right)^{\prime \prime}=V^{\left(v_{1}\right)}(x)+2\left(\log \psi_{\nu_{1}}(x)\right)^{\prime \prime}=V(x)
$$


and the final potential coincides with the initial potential. The image of the eigenfunction $\psi_{\lambda}$, $\lambda \neq v_{1}$, is given by

$$
\begin{aligned}
\psi_{\lambda}^{\left(\nu_{1}, \nu_{1}\right)}(x) & =\frac{W\left(\psi_{\lambda}^{\left(\nu_{1}\right)}, \psi_{\nu_{1}}^{\left(\nu_{1}\right)} \mid x\right)}{\psi_{\nu_{1}}^{\left(\nu_{1}\right)}(x)} \sim \frac{W\left(W\left(\psi_{\lambda}, \psi_{\nu_{1}}\right) / \psi_{\nu_{1}}, 1 / \psi_{\nu_{1}} \mid x\right)}{1 / \psi_{\nu_{1}}(x)} \\
& \sim \frac{W^{\prime}\left(\psi_{\lambda}, \psi_{\nu_{1}} \mid x\right)}{\psi_{\nu_{1}}(x)},
\end{aligned}
$$

that is, using the Wronskian theorem

$$
\psi_{\lambda}^{\left(\nu_{1}, \nu_{1}\right)}(x) \sim \psi_{\lambda}(x) .
$$

As for the image of $\psi_{\nu_{1}}$, it is

$$
\psi_{v_{1}}^{\left(\nu_{1}, \nu_{1}\right)}(x) \sim \frac{1}{\psi_{\nu_{1}}^{\left(\nu_{1}\right)}(x)} \sim \psi_{\nu_{1}}(x) .
$$

These results prove that, in the two-step case, using the same seed function twice in a chain of DBT gives the identity transformation. As expected intuitively, this result is generalizable to higher order chains. Namely, we have

Lemma 2. By completing a chain by state-deleting DBT associated to some of the precedingly used seed functions we obtain the images of a reduced chain of $(m-k)$ steps where these seed functions associated tov $v_{i_{1}}, \ldots, v_{i_{k}}$ have been suppressed

$$
\left\{\begin{array}{l}
V^{\left(N_{m}, v_{i_{1}}, \ldots, v_{i_{k}}\right)}(x)=V^{\left(N_{m} / v_{i_{1}}, \ldots, v_{i_{k}}\right)}(x), \\
\psi_{\lambda}^{\left(N_{m}, v_{i_{1}}, \ldots, v_{i_{k}}\right)}(x) \sim \psi_{\lambda}^{\left(N_{m} / v_{i_{1}}, \ldots, v_{i_{k}}\right)}(x) .
\end{array}\right.
$$

Proof. Consider first a three step case $m=3$ where $N_{2}=\left(v_{1}, v_{2}\right) N_{3}=\left(N_{2}, v_{1}\right)$. We have from Eqs. (8) and (12)

$$
V^{\left(N_{3}\right)}(x) V(x)-2\left(\log W\left(\psi_{\nu_{1}}, \psi_{\nu_{2}} \mid x\right) \psi_{\nu_{1}}^{\left(N_{2}\right)}(x)\right)^{\prime \prime} .
$$

Since (cf. Lemma 1)

$$
\psi_{\nu_{1}}^{\left(N_{2}\right)}(x) \sim \frac{\psi_{\nu_{2}}(x)}{W\left(\psi_{\nu_{1}}, \psi_{\nu_{2}} \mid x\right)},
$$

we obtain

$$
V^{\left(N_{3}\right)}(x)=V^{\left(\nu_{2}\right)}(x) .
$$

For $\lambda \neq v_{1}, v_{2}$, we have, using Lemma 1 and the standard Crum formula

$$
\begin{aligned}
\psi_{\lambda}^{\left(N_{3}\right)}(x) & =\frac{W\left(\psi_{\lambda}^{\left(N_{2}\right)}, \psi_{\nu_{1}}^{\left(N_{2}\right)} \mid x\right)}{\psi_{\nu_{1}}^{\left(N_{2}\right)}(x)}=\frac{W\left(\psi_{\lambda}^{\left(\nu_{2}, \nu_{1}\right)}, \psi_{\nu_{1}}^{\left(\nu_{2}, \nu_{1}\right)} \mid x\right)}{\psi_{\nu_{1}}^{\left(\nu_{2}, \nu_{1}\right)}(x)} \\
& \sim \frac{W\left(W\left(\psi_{\lambda}^{\left(\nu_{2}\right)}, \psi_{\nu_{1}}^{\left(\nu_{2}\right)}\right) / \psi_{\nu_{1}}^{\left(\nu_{2}\right)}, 1 / \psi_{\nu_{1}}^{\left(\nu_{2}\right)} \mid x\right)}{1 / \psi_{\nu_{1}}^{\left(\nu_{2}\right)}(x)} \sim \frac{W^{\prime}\left(\psi_{\lambda}^{\left(\nu_{2}\right)}, \psi_{\nu_{1}}^{\left(\nu_{2}\right)} \mid x\right)}{\psi_{\nu_{1}}^{\left(\nu_{2}\right)}(x)} .
\end{aligned}
$$

With the Wronskian theorem, we then obtain

$$
\psi_{\lambda}^{\left(\nu_{1}, v_{2}, v_{1}\right)}(x) \sim \psi_{\lambda}^{\left(\nu_{2}\right)}(x) .
$$

We can also note that, in a coherent way, using Eq. (35) we can write

$$
\psi_{\lambda}^{\left(\nu_{1}, \nu_{1}, \nu_{2}\right)}(x)=\frac{W\left(\psi_{\lambda}^{\left(\nu_{1}, \nu_{1}\right)}, \psi_{\nu_{2}}^{\left(\nu_{1}, \nu_{1}\right)} \mid x\right)}{\psi_{\nu_{2}}^{\left(\nu_{1}, \nu_{1}\right)}(x)}=\frac{W\left(\psi_{\lambda}, \psi_{\nu_{2}} \mid x\right)}{\psi_{\nu_{2}}(x)}=\psi_{\lambda}^{\left(\nu_{2}\right)}(x)
$$


and with Lemma 1

$$
\psi_{\lambda}^{\left(\nu_{2}, \nu_{1}, \nu_{1}\right)}(x)=\frac{W\left(\psi_{\lambda}^{\left(\nu_{2}, \nu_{1}\right)}, \psi_{\nu_{1}}^{\left(\nu_{2}, \nu_{1}\right)} \mid x\right)}{\psi_{\nu_{1}}^{\left(\nu_{2}, \nu_{1}\right)}(x)}=\frac{W\left(\psi_{\lambda}^{\left(\nu_{1}, \nu_{2}\right)}, \psi_{\nu_{1}}^{\left(\nu_{1}, \nu_{2}\right)} \mid x\right)}{\psi_{\nu_{1}}^{\left(\nu_{1}, \nu_{2}\right)}(x)}=\psi_{\lambda}^{\left(N_{3}\right)}(x)=\psi_{\lambda}^{\left(\nu_{2}\right)}(x)
$$

It remains to consider the cases $\lambda=v_{1}$ and $\lambda=v_{2}$. In the first case, we have

$$
\psi_{\nu_{1}}^{\left(N_{3}\right)}(x) \sim \frac{1}{\psi_{\nu_{1}}^{\left(N_{2}\right)}(x)} \sim \psi_{\nu_{1}}^{\left(v_{2}\right)}(x)=\frac{\psi_{\nu_{2}}(x)}{W\left(\psi_{\nu_{1}}, \psi_{\nu_{2}} \mid x\right)}
$$

and in the second case

$$
\begin{aligned}
\psi_{v_{2}}^{\left(N_{3}\right)}(x) & =\frac{W\left(\psi_{v_{2}}^{\left(N_{2}\right)}, \psi_{v_{1}}^{\left(N_{2}\right)} \mid x\right)}{\psi_{v_{1}}^{\left(N_{2}\right)}(x)} \sim \frac{W\left(1 / \psi_{v_{2}}^{\left(\nu_{1}\right)}, 1 / \psi_{\nu_{1}}^{\left(\nu_{2}\right)} \mid x\right)}{1 / \psi_{\nu_{1}}^{\left(\nu_{2}\right)}(x)} \\
& \sim \frac{W\left(\frac{\psi_{v_{1}}}{W\left(\psi_{v_{2}}, \psi_{v_{1}}\right)}, \frac{\psi_{v_{2}}}{W\left(\psi_{v_{1}}, \psi_{v_{2}}\right)} \mid x\right)}{\frac{\psi_{v_{2}}(x)}{W\left(\psi_{v_{1}}, \psi_{v_{2}} \mid x\right)}},
\end{aligned}
$$

that is,

$$
\psi_{v_{2}}^{\left(N_{3}\right)}(x) \sim \frac{1}{\psi_{v_{2}}(x)} \sim \psi_{v_{2}}^{\left(v_{2}\right)}(x) .
$$

The lemma is then verified in the 3-step case.

Consider now the general case $N_{m}=\left(v_{1}, \ldots, v_{m}\right)$ and apply to $V^{\left(N_{m}\right)}$ the DBT $A\left(w_{v_{i_{1}}}^{\left(N_{m}\right)}\right)$. The resulting potential is

$$
\begin{aligned}
V^{\left(N_{m}, v_{i_{1}}\right)}(x) & =V^{\left(N_{m}\right)}(x)-2\left(\log \left(\psi_{v_{i_{1}}}^{\left(N_{m}\right)}(x)\right)\right)^{\prime \prime}=V-2\left(\log \left(W^{\left(N_{m}\right)}(x) \psi_{v_{i_{1}}}^{\left(N_{m}\right)}(x)\right)\right)^{\prime \prime} \\
& =V(x)-2\left(\log \left(W^{\left(N_{m} / v_{i_{1}}\right)}(x)\right)\right)^{\prime \prime}=V^{\left(N_{m} / v_{i_{1}}\right)}(x) .
\end{aligned}
$$

The recurrence is immediate and if we apply a chain of $k$ DBT based on the seeds functions $\psi_{v_{i_{1}}}^{\left(N_{m}\right)}, \ldots, \psi_{v_{i_{k}}}^{\left(N_{m}\right)}$ of $V^{\left(N_{m}\right)}, 1 \leq i_{1}<\ldots<i_{k} \leq m$, the final extended potential can be written as

$$
V^{\left(N_{m}, v_{i_{1}}, \ldots, v_{i_{k}}\right)}(x)=V^{\left(N_{m} / v_{i_{1}}, \ldots, v_{i_{k}}\right)}(x) .
$$

We know that $\psi_{\lambda}^{\left(N_{m} / v_{i_{1}}, \ldots, v_{i_{k}}\right)}(x)$ is an eigenfunction of $V^{\left(N_{m}, v_{i_{1}}, \ldots, v_{i_{k}}\right)}(x)$ for the eigenvalue $E_{\lambda}$ but we have still to verify that it can be identified with $\psi_{\lambda}^{\left(N_{m}, v_{i_{1}}, \ldots, v_{i_{k}}\right)}(x)$.

Consider first the case $\lambda=v_{j} \in N_{m}$. The image of $\psi_{v_{j}}^{\left(N_{m}\right)}$, where $1 \leq j \leq m$, by the DBT $A\left(\psi_{v_{i_{1}}}^{\left(N_{m}\right)}\right), j \neq i_{1}$, is

$$
\psi_{v_{j}}^{\left(N_{m}, v_{i_{1}}\right)}(x)=\frac{W\left(\psi_{v_{j}}^{\left(N_{m}\right)}, \psi_{v_{i_{1}}}^{\left(N_{m}\right)} \mid x\right)}{\psi_{v_{i_{1}}}^{\left(N_{m}\right)}(x)} .
$$

Using Lemma 1, it can be rewritten as

$$
\psi_{v_{j}}^{\left(N_{m}, v_{i_{1}}\right)}(x) \sim \frac{W\left(W^{\left(N_{m} / v_{j}\right)}, W^{\left(N_{m} / v_{i_{1}}\right)} \mid x\right)}{W^{\left(N_{m}\right)}(x) W^{\left(N_{m} / v_{i_{1}}\right)}(x)}
$$

and with the Jacobi identity for Wronskians ${ }^{55}$

$$
W^{\left(N_{m}\right)}(x)=\frac{W\left(W^{\left(N_{m} / v_{j}\right)}, W^{\left(N_{m} / v_{i_{1}}\right)} \mid x\right)}{W^{\left(N_{m} / v_{j}, v_{i_{1}}\right)}(x)},
$$

we obtain

$$
\psi_{v_{j}}^{\left(N_{m}, v_{i_{1}}\right)}(x) \sim \frac{W^{\left(N_{m} / v_{j}, v_{i_{1}}\right)}(x)}{W^{\left(N_{m} / v_{i_{1}}\right)}(x)} \sim \psi_{v_{j}}^{\left(N_{m} / v_{i_{1}}\right)}(x) .
$$


The generalization is immediate and we have for $j \neq i_{1}, \ldots, i_{k}$

$$
\psi_{v_{j}}^{\left(N_{m}, v_{i_{1}}, \ldots, v_{i_{k}}\right)}(x) \sim \frac{W^{\left(N_{m} / v_{j}, v_{i_{1}}, \ldots, v_{i_{k}}\right)}(x)}{W^{\left(N_{m} / v_{i_{1}}, \ldots, v_{i_{k}}\right)}(x)} \sim \psi_{v_{j}}^{\left(N_{m} / v_{i_{1}}, \ldots, v_{i_{k}}\right)}(x) .
$$

In the case where $\lambda \notin N_{m}$, using Lemma 1 and applying the DBT $A\left(\psi_{v_{i_{1}}}^{\left(N_{m}\right)}\right)$ to $\psi_{\lambda}^{\left(N_{m}\right)}(x)$, we deduce

$\psi_{\lambda}^{\left(N_{m}, v_{i_{1}}\right)}(x)=\frac{W\left(\psi_{\lambda}^{\left(N_{m}\right)}, \psi_{v_{i_{1}}}^{\left(N_{m}\right)} \mid x\right)}{\psi_{v_{i_{1}}}^{\left(N_{m}\right)}(x)} \sim \frac{W\left(W\left(\psi_{\lambda}^{\left(N_{m} / v_{i_{1}}\right)}, \psi_{v_{i_{1}}}^{\left(N_{m} / v_{i_{1}}\right)}\right) / \psi_{v_{i_{1}}}^{\left(N_{m} / v_{i_{1}}\right)}, 1 / \psi_{v_{i_{1}}}^{\left(N_{m} / v_{i_{1}}\right)} \mid x\right)}{1 / \psi_{v_{i_{1}}}^{\left(N_{m} / v_{i_{1}}\right)}(x)}$,

that is, using the Wronskian theorem

$$
\psi_{\lambda}^{\left(N_{m}, v_{i_{1}}\right)}(x) \sim \frac{W^{\prime}\left(\psi_{\lambda}^{\left(N_{m} / v_{i_{1}}\right)}, \psi_{v_{i_{1}}}^{\left(N_{m} / v_{i_{1}}\right)} \mid x\right)}{\psi_{v_{i_{1}}}^{\left(N_{m} / v_{i_{1}}\right)}(x)} \sim \psi_{\lambda}^{\left(N_{m} / v_{i_{1}}\right)}(x) .
$$

Here, again the generalization is immediate and we can write

$$
\psi_{\lambda}^{\left(N_{m}, v_{i_{1}}, \ldots, v_{i_{k}}\right)}(x) \sim \psi_{\lambda}^{\left(N_{m} / v_{i_{1}}, \ldots, v_{i_{k}}\right)}(x),
$$

which achieves the proof of Lemma 2.

\section{DISCONJUGACY AND REGULAR EXTENSIONS}

From $V$, the DBT $A\left(w_{v}\right)$ generates a new potential $V^{(v)}$, the eigenfunctions of which being directly obtained from those of $V$ via Eq. (6). Nevertheless, in general $w_{\nu}(x)$ and then the transformed potential $V^{(v)}(x)$ are singular at the nodes of $\psi_{v}(x)$. For instance, if $\psi_{n}(x)(v=n)$ is a bound state of $\widehat{H}$, the Sturm oscillation theorem ${ }^{43,45}$ implies that $V^{(n)}$ is regular only when $n=0$, that is, when the seed function is the ground state of $\widehat{H}$. This corresponds exactly to the usual SUSY partnership in quantum mechanics. ${ }^{38,39}$

We can however envisage the use of a seed function that is any other regular solution of Eq. (2) as long as it has no zero on the considered real interval $I$ and as the transformed eigenfunction satisfies the required boundary conditions, even if this seed function does not correspond to a physical state.

To control the regularity of $w_{\nu}$, we can make use of the disconjugacy properties of the Schrödinger equation for negative eigenvalues. In the SUSY QM frame, this possibility has been first envisaged by Sukumar. ${ }^{41}$

A second order differential equation like Eq. (1) is said to be disconjugated on $I \subset \mathbb{R}(V(x)$ is supposed to be continuous on $I$ ) if every solution of this equation has at most one zero on $I .^{43,44,46}$ As it is well known, this zero is necessarily simple and at this value the considered solution changes its sign. For a closed or open interval $I$, the disconjugacy of Eq. (1) is equivalent to the existence of solutions of this equation which are everywhere non-zero on $I .43,44,46$

We have also the following result:

Disconjugacy theorem: ${ }^{43,44}$ If there exists a continuously differentiable solution on Iof the Riccati inequation

$$
-w^{\prime}(x)+w^{2}(x) \leq G(x)
$$

then the equation

$$
\psi^{\prime \prime}(x)-G(x) \psi(x)=0
$$

is disconjugated on $I$.

In our case Eq. (59) is the Schrödinger equation (1) with $G(x)=V(x)-E_{\lambda}$ and if $E_{\lambda} \leq E_{0}$ $=0$, we have

$$
-w_{0}^{\prime}(x)+w_{0}^{2}(x) \leq V(x)-E_{\lambda},
$$


$w_{0}(x)$ being continuously differentiable on $I$. The above theorem ensures the existence of nodeless solutions of Eq. (1) for the eigenvalue $E_{\lambda}$. To prove that a given solution $\phi(x)$ belongs to this category, it is sufficient to determine the signs of the boundaries of $I$. If they are identical, then $\phi$ is nodeless and if they are opposite, then $\phi$ presents a unique zero on $I$. In the first case, $V(x)+2 v^{\prime}(x)$, where $v(x)=-\phi^{\prime}(x) / \phi(x)$, constitutes a regular (quasi)isospectral extension of $V(x)$. The disconjugacy sector of Eq. (1) corresponds then to the values of the spectral parameter $E_{\lambda} \leq E_{0}$.

\section{REGULAR CHAINS OF DBT AND KREIN-ADLER THEOREM}

The question of the link between the regularity of the successive extensions obtained by a chain of DBT and the choice of the associated seed functions is natural. In his seminal paper, Crum ${ }^{2,59}$ was the first to establish that if we take a sequence $N_{m}=(0, \ldots, m-1)=M$ corresponding to seed functions which are consecutive eigenstates starting from the fundamental one and that we call a complete chain of DBT, then

$$
V^{(M)}(x)=V(x)+2\left(\log W\left(\psi_{0}, \ldots, \psi_{m-1} \mid x\right)\right)^{\prime \prime}
$$

is regular and its spectrum is given by

$$
\left\{\begin{array}{c}
E_{k}^{(M)}=E_{k+m} \\
\phi_{k}^{(M)}(x)=\psi_{k+m}^{(M)}(x)=\frac{W\left(\psi_{0}, \ldots, \psi_{m-1}, \psi_{k} \mid x\right)}{W\left(\psi_{0}, \ldots, \psi_{m-1} \mid x\right)}, \quad k \geq 0 .
\end{array}\right.
$$

This means that although each of the $\psi_{i}, 1 \leq i \leq m-1$, has exactly $i$ nodes, the Wronskian of the family $\left(\psi_{0}, \ldots, \psi_{m-1}\right)$ is, as for it, free of zero.

It can be very easily understood by noting that the action on the spectrum of the successive DBT based on such eigenstates suppresses at each step the level associated to the used seed function. Such DBT are said to be state-deleting. In this perspective, since $\psi_{j}^{\left(N_{j}\right)}(x), N_{j}=(0, \ldots, j-1)$, is the fundamental eigenstate of $V^{\left(N_{j}\right)}(x)$, the chain can be viewed as a succession of SUSY QM partnerships and all the extensions along the chain are regular. This is exactly the argument at the basis of the construction of hamiltonians hierarchy in SUSY QM. ${ }^{38,39}$

$\mathrm{Krein}^{57}$ and later Adler ${ }^{58}$ have enlarged this regularity property to lacunary chains of statedeleting DBT.

Krein-Adler theorem: Consider a chain of DBT characterized by the m-uple $N_{m}=\left(n_{1}, \ldots\right.$, $\left.n_{m}\right)$ of positive integers such that the corresponding seed functions $\psi_{n_{i}}$ are eigenstates of the initial potential.

The extended potential $V^{\left(N_{m}\right)}(x)$ is regular iff the sequence $N_{m}$ is constituted by fragments of an even number of consecutive positive integer, except the first one which is of arbitrary length. In other words, the spectrum of the final extension $V^{\left(N_{m}\right)}(x)$ must contain only even gaps (gaps constituted by an even number of consecutive missing levels). This condition is equivalently described by the Krein condition

$$
\left(n-n_{1}\right)\left(n-n_{2}\right) \ldots\left(n-n_{m}\right) \geq 0, \forall n \in N
$$

Such a lacunary chain is said of the Krein-Adler type.

In Ref. 67, Samsonov extended the Krein-Adler result still further, showing that it is possible to employ sets of two juxtaposed eigenfunctions whose associated eigenvalues are taken between two consecutive energy levels. These results have been used extensively in the context of higher order SUSY (see, for instance, Refs. 41 and 60-71).

Recently, we have proven that for the specific class of translationally shape invariant potentials of the second category, it is possible to build chains of regular DBT of arbitrary length by using eigenfunctions in the disconjugacy sector ${ }^{9,31,50}$ associated to exceptional Laguerre or Jacobi polynomials. 


\section{TSIP}

Consider a potential $V(x ; \alpha)$ which depends upon a (multi)parameter $\alpha \in \mathbb{R}^{N}$ and with a (finite or infinite) bound state spectrum $\left(E_{n}, \psi_{n}\right)_{n>0}$, the ground level being supposed to be zero: $E_{0}(\alpha)$ $=0$. In the framework of SUSY QM, such a potential is said to be shape invariant (SIP) ${ }^{38-40}$ if its SUSY partner

$$
V^{(0)}(x ; \alpha)=V(x ; \alpha)+2 w_{0}^{\prime}(x ; \alpha),
$$

keeps the same functional form as the initial potential. Namely,

$$
V^{(0)}(x ; \alpha)=V(x ; f(\alpha))+R(\alpha),
$$

$R(\alpha) \in \mathbb{R}$ and $f(\alpha) \in \mathbb{R}^{N}$ being two given functions of $\alpha$.

In this case, it can be shown ${ }^{38-40}$ that the complete bound state energy spectrum of $\widehat{H}(\alpha)$ $=-\frac{d^{2}}{d x^{2}}+V(x ; \alpha)$ is given by

$$
E_{n}(\alpha)=\sum_{k=0}^{n-1} R\left(\alpha_{k}\right),
$$

where $\alpha_{k}=f^{(k)}(\alpha)=\overbrace{f \circ \ldots \circ f}^{\mathrm{k} \text { times }}(\alpha)$.

As for the corresponding eigenstates, they can be written as

$$
\psi_{n}(x ; \alpha) \sim \widehat{A}^{+}(\alpha) \psi_{n-1}\left(x ; \alpha_{1}\right) \sim \widehat{A}^{+}(\alpha) \ldots \widehat{A}^{+}\left(\alpha_{n-1}\right) \psi_{0}\left(x ; \alpha_{n}\right),
$$

where $\widehat{A}^{+}(\alpha)=-\frac{d}{d x}+w_{0}(x ; \alpha)$. For the corresponding RS functions, the above differential relation becomes an algebraic one

$$
w_{n}(x ; \alpha)=w_{0}(x ; \alpha)-\frac{E_{n}(\alpha)}{w_{0}(x ; \alpha)+w_{n-1}\left(x ; \alpha_{1}\right)},
$$

which allows to write the excited states as terminating continued fractions in terms of $w_{0}{ }^{32}$

When $f$ is a simple translation $f(\alpha)=\alpha+\varepsilon, \varepsilon=\left(\varepsilon^{(1)}, \ldots, \varepsilon^{(N)}\right) \in \mathbb{R}^{N}, V$ is said to be TSIP. Without loss of generality, we can take $\varepsilon^{(k)}= \pm 1$ or 0 . For all the known TSIP, we have $\alpha \in \mathbb{R}$ (first category TSIP) or $\alpha=\left(\alpha^{(1)}, \alpha^{(2)}\right) \in \mathbb{R}^{2}$ (second category TSIP). ${ }^{32,38,39}$

Since $R(\alpha)=E_{1}(\alpha)$, Eq. (66) can be rewritten as

$$
E_{n}(\alpha)=\sum_{i=0}^{n-1} E_{1}\left(\alpha_{i}\right)
$$

and the dispersion relation, that is, $E_{n}(\alpha)$ as a function of $n$ and $\alpha$, satisfies then the characteristic identity

$$
E_{n+m}(\alpha)-E_{m}(\alpha)=E_{n}\left(\alpha_{m}\right),
$$

which can be used to extend the dispersion relation to all integer values of $n$. We easily verify that this identity is satisfied as soon as $E_{n}(\alpha)$ is of the form

$$
E_{n}(\alpha)= \pm\left(g(\alpha)-g\left(\alpha_{n}\right)\right)
$$

$g(\alpha)$ being an arbitrary function of $\alpha$.

The set of TSIP contains all the potentials classically known to be exactly solvable, i.e., for which we know explicitly the dispersion relation and whose eigenfunctions can be expressed in closed analytical form in terms of elementary transcendental functions, namely, the harmonic, isotonic, Morse, Kepler-Coulomb, Eckart, Darboux-Pöschl-Teller hyperbolic and trigonometric, and RosenMorse hyperbolic and trigonometric potentials. Until recently it was commonly believed that these potentials were the only ones to possess the translational shape invariance property. However, it has been shown recently that they are in fact only primary TSIP (PTSIP) from which it is possible in some cases to build infinite towers of secondary TSIP (STSIP) which are extensions of the previous ones and which share the same translational shape invariance properties. ${ }^{31}$ 
An important feature of the PTSIP is that their eigenfunctions $\psi_{n}$ are equal, up to a gauge factor, to classical orthogonal polynomials in an appropriate variable $z$ (which can be $n$ dependent) and we call $\psi_{n}$ a quasi-polynomial in this variable. The associated RS functions are rational functions $z \cdot{ }^{32}$ As for the function defined above, it is given by $g(\alpha)=\alpha^{2}$ or $g(\alpha)=1 / \alpha^{2} .{ }^{32}$

Note that in general, the range of values of the multiparameter $\alpha$ is subject to limiting constraints of the type $\alpha \in U \subset \mathbb{R}^{N}$, that is, $\alpha^{(i)} \in U^{(i)}, \quad U^{(i)} \subset \mathbb{R}$, in order to have a appropriate regular behaviour for $V(x ; \alpha)$ and to ensure that the $\psi_{n}(x ; \alpha)$ satisfy the required Dirichlet boundary conditions.

\section{DISCRETE SYMMETRIES, RATIONAL EXTENSIONS OF TSIP, AND STATE-ADDING DBT}

The primary TSIP potentials possess specific discrete symmetries $\Gamma_{i}$ which act in the space of parameters and which are covariance transformations for the considered potential

$$
\left\{\begin{array}{c}
\alpha \stackrel{\Gamma_{i}}{\rightarrow} \alpha_{i} \\
V(x ; \alpha) \stackrel{\Gamma_{i}}{\rightarrow} V\left(x ; \alpha_{i}\right)=V(x ; \alpha)+\delta_{i}(\alpha) .
\end{array}\right.
$$

In the space of parameters (which is two-dimensional for the second category and onedimensional for the first), the $\Gamma_{i}$ correspond to the reflections with respect to the coordinate axis. For the second category potentials (for which the parameter is two-dimensional), we then have three different symmetries $\Gamma_{+}, \Gamma_{-}$and $\Gamma_{3}=\Gamma_{+} \circ \Gamma_{-}$, while for the first category ones (for which the parameter is one-dimensional) we have only one symmetry $\Gamma_{3}$.

$\Gamma_{i}$ transforms an excited eigenstate $\psi_{n}$ into a unphysical RS function $\phi_{n, i}(x ; \alpha)=\Gamma_{i}\left(\psi_{n}(x ;\right.$ $\alpha)$ ) in the disconjugacy sector of $V(x ; \alpha)$, that is, associated to the negative eigenvalue $\mathcal{E}_{n, i}(\alpha)$ $=\Gamma_{i}\left(E_{n}(\alpha)\right)=E_{n}\left(\alpha_{i}\right)-\delta_{i}(\alpha)<0$.

The $\Gamma_{i}$ symmetries preserve the functional structure of the initial eigenstates and the $\phi_{n, i}$ are then also quasi-polynomials with rational associated RS functions $v_{n, i}(x ; \alpha)$.

If the transformed RS function $v_{n, i}$ is regular on $I$, it can be used to build a regular extended potential (see Eqs. (5) and (6))

$$
V^{(n, i)}(x ; \alpha)=V(x ; \alpha)+2 v_{n, i}^{\prime}(x ; \alpha)
$$

(quasi)isospectral to $V(x ; \alpha)$ that we call a rational extension of $V$.

To the $\psi_{k \geq 0}$ correspond eigenstates of $V^{(n, i)}$ which are given by (see Eq. (3))

$$
\left\{\begin{array}{l}
w_{k}^{(n, i)}(x ; \alpha)=-v_{n, i}(x ; \alpha)+\left(E_{k}(\alpha)-\mathcal{E}_{n, i}(\alpha)\right) /\left(v_{n, i}(x ; \alpha)-w_{k}(x ; \alpha)\right), \\
\psi_{k}^{(n, i)}(x ; \alpha)=\exp \left(-\int d x w_{k}^{(n, i)}(x ; \alpha)\right) \sim W\left(\phi_{n, i}, \psi_{k} \mid x\right) / \phi_{n, i}(x ; \alpha),
\end{array}\right.
$$

for the respective energies $E_{k}(\alpha)$.

The nature of the isospectrality depends on whether $1 / \phi_{n, i}(x ; \alpha)$ satisfies the appropriate Dirichlet boundary conditions. If it is the case, then $1 / \phi_{n, i}(x ; \alpha)$ is a physical eigenstate of $\widehat{H}^{(n, i)}(\alpha)$ $=-d^{2} / d x^{2}+V^{(n, i)}(x ; \alpha)$ for the eigenvalue $\mathcal{E}_{n, i}(\alpha)$ and we only have quasi-isospectrality (or essential isospectrality) between $V(x ; \alpha)$ and $V^{(n, i)}(x ; \alpha)$, the spectrum of $V^{(n, i)}$ containing a supplementary level $\mathcal{E}_{n, i}(\alpha)$ below $E_{0}=0$. The DBT $A\left(v_{n, i}\right)$ is then called a state-adding DBT. If it is not the case, the isospectrality between $V^{(n, i)}(x ; \alpha)$ and $V(x ; \alpha)$ is strict.

It has been proven ${ }^{29}$ that the regular $V^{(n,+)}$ and $V^{(n,-)}$ are isospectral to the initial potential while the regular $V^{(n, 3)}$ are only quasi-isospectral to $V$. Moreover, the regular extensions $V^{(n, \pm)}$ inherit the shape invariance properties of the initial potential while the regular $V^{(n, 3)}$ do not. The DBT $A\left(v_{n, 3}\right)$ is in fact a reciprocal SUSY QM partnership

$$
A\left(v_{n, 3}\right)=A^{-1}\left(w_{0}^{(n)}\right)
$$


In the following, we consider specifically the extensions associated to the $\Gamma_{3}$ symmetry which acts as

$$
\left\{\begin{array}{c}
\Gamma_{3}(V(x ; \alpha))=V(x ;-\alpha)=V(x ; \alpha)+\delta(\alpha), \\
\Gamma_{3}\left(\psi_{n}(x ; \alpha)\right)=\psi_{n}(x ;-\alpha)=\phi_{n, 3}(x ; \alpha),
\end{array}\right.
$$

(if $\left(\alpha^{(1)}, \alpha^{(2)}\right)=(\alpha, \beta)$, then $-\alpha=\left(-\alpha^{(1)},-\alpha^{(2)}\right)$ and we note $\delta(\alpha)$ for $\left.\delta_{3}(\alpha)\right)$.

For all the PTSIP, we have

$$
\mathcal{E}_{n, 3}(\alpha)=E_{-(n+1)}(\alpha)<0
$$

and $\phi_{n, 3}(x ; \alpha)$ diverges at both limits of the definition domain (which implies that $1 / \phi_{n, 3}$ satisfies the required Dirichlet boundary conditions).

This leads us to fix

$$
\psi_{-(n+1)}(x ; \alpha)=\phi_{n, 3}(x ; \alpha) .
$$

This also implies the identity $\left(E_{0}(\alpha)=0\right)$

$$
\delta(a)=E_{-(n+1)}(\alpha)-E_{n}(\alpha)=E_{-1}(\alpha),
$$

with (see Eq. (70))

$$
E_{-1}(\alpha)=-E_{1}\left(\alpha_{-1}\right) .
$$

Since $\psi_{-(n+1)}$ is in the disconjugacy sector of $V(x ; \alpha)$, the disconjugacy theorem implies that it is free of nodes only if it has the same sign at the boundaries of the definition interval, which is the case when $\alpha_{n} \in U$ and $n$ is odd.

\section{GENERALIZED KREIN-ADLER THEOREM FOR TSIP}

\section{A. Complete chains of state-adding DBT for TSIP}

For the TSIP, we can envisage the construction of complete chains of DBT based not only on successive state-deleting DBT but also complete chains of DBT based on state-adding DBT. These chains, characterized by $m$-uple of the type $-M=(-1, \ldots,-m)$, correspond to several successive reversed SUSY partnerships and are "mirror images" of the chains of state-deleting DBT associated to the sequence $M=(0, \ldots, m-1)$. The extended potentials thus generated also satisfy a reversed shape invariance condition.

Lemma 3. Suppose that TSIP $V(x ; \alpha)$ admits a discrete $\Gamma_{3}$ symmetry such that $\Gamma_{3}\left(\psi_{n}(x ; \alpha)\right)$ $=\psi_{-(n+1)}(x ; \alpha)$. If $\alpha_{-1} \in A$, the action of the DBT $A\left(w_{-1}\right)$ on $V(x ; \alpha)$ is a reversed SUSY partnership and $V(x ; \alpha)$ satisfies a reversed shape invariance condition

$$
V^{(-1)}(x ; \alpha)=V\left(x ; \alpha_{-1}\right)+E_{-1}(\alpha) .
$$

When $A\left(w_{-1}\right)$ is state-adding, the spectrum of the regular extended potential $V^{(-1)}(x ; \alpha)$ is given by

$$
\left\{\begin{aligned}
E_{j}^{(-1)}(\alpha) & =E_{j-1}(\alpha) \\
\phi_{0}^{(-1)}(x ; \alpha)=\psi_{-1}^{(-1)}(x ; \alpha) & \sim 1 / \psi_{-1}(x ; \alpha) \sim \psi_{0}\left(x ; \alpha_{-1}\right), \quad j \geq 0 . \\
\phi_{j+1}^{(-1)}(x ; \alpha)=\psi_{j}^{(-1)}(x ; \alpha) & \sim \frac{W\left(\psi_{j}, \psi_{-1} \mid x\right)}{\psi_{-1}(x ; \alpha)} \sim \psi_{j+1}\left(x ; \alpha_{-1}\right)
\end{aligned}\right.
$$

Proof: Acting on $V(x ; \alpha)$ with the regular DBT $A\left(w_{-1}\right)=A\left(v_{0,3}\right)$ and using Eqs. (76) and (79), we have

$$
\begin{aligned}
V^{(-1)}(x ; \alpha) & =V(x ; \alpha)+2 w_{-1}^{\prime}(x ; \alpha)=V(x ;-\alpha)-E_{-1}(\alpha)+2 w_{0}^{\prime}(x ;-\alpha) \\
& =V^{(0)}(x ;-\alpha)-E_{-1}(\alpha) .
\end{aligned}
$$


Applying the translational shape invariance property (see Eq. (65) of $V$ ), we obtain $V^{(-1)}(x ; \alpha)=V\left(x ;(-\alpha)_{1}\right)+E_{1}(-\alpha)-E_{-1}(\alpha)=V\left(x ;-\alpha_{-1}\right)+E_{1}(-\alpha)-E_{-1}(\alpha)$,

or (see Eq. (76))

$$
V^{(-1)}(x ; \alpha)=V\left(x ; \alpha_{-1}\right)-S(\alpha)
$$

with

$$
S(\alpha)=E_{-1}(\alpha)-E_{-1}\left(\alpha_{-1}\right)-E_{1}(-\alpha)
$$

Equation (85) ensures the regularity of $V^{(-1)}$ when $\alpha_{-1} \in U$. It is a shape invariance condition for the reversed SUSY partnership corresponding to the DBT $A\left(w_{-1}\right)$ based on the "symmetrized ground state" $\psi_{-1}=\Gamma_{3}\left(\psi_{0}\right)$.

If $A\left(w_{-1}\right)$ is a state-adding DBT, the ground level of $V^{(-1)}$ is $E_{-1}(\alpha)$ with a fundamental eigenstate

$$
\phi_{0}^{(-1)}(x ; \alpha)=\psi_{-1}^{(-1)}(x ; \alpha)=1 / \psi_{-1}(x ; \alpha) .
$$

But the ground level of $V\left(x ; \alpha_{-1}\right)-S(\alpha)$ is $E_{0}\left(\alpha_{-1}\right)-S(\alpha)=-S(\alpha)$ with the corresponding eigenstate $\psi_{0}\left(x ; \alpha_{-1}\right)$ which (when $\left.\alpha_{-1} \in A\right)$ is supposed to satisfy the required Dirichlet boundary conditions. We then deduce

$$
\left\{\begin{array}{c}
S(\alpha)=-E_{-1}(\alpha) \geq 0 \\
\psi_{-1}^{(-1)}(x ; \alpha) \sim \psi_{0}\left(x ; \alpha_{-1}\right) \sim 1 / \psi_{-1}(x ; \alpha) .
\end{array}\right.
$$

As for the excited eigenstates $\phi_{j+1}^{(-1)}$ of this extension, associated to the eigenvalues $E_{j \geq 0}(\alpha)$, they are given by the usual Darboux-Crum formula (8)

$$
\phi_{j+1}^{(-1)}(x ; \alpha)=\psi_{j}^{(-1)}(x ; \alpha) \sim \frac{W\left(\psi_{j}, \psi_{-1} \mid x\right)}{\psi_{-1}(x ; \alpha)} .
$$

Using Eqs. (85) and (70), we see that $\psi_{j}^{(-1)}(x ; \alpha)$ is also an eigenstate of $\widehat{H}\left(\alpha_{-1}\right)$ for the eigenvalue $E_{j+1}(\alpha)$, which leads to

$$
\phi_{j+1}^{(-1)}(x ; \alpha) \sim \psi_{j+1}\left(x ; \alpha_{-1}\right) .
$$

The action of the reversed SUSY partnership $A\left(\psi_{-1}\right)$ is completely analogous to the usual SUSY partnership but with an opposite shift of the parameter and the addition of a new ground level rather than the suppression of the original one. We can then envisage the construction of a complete chain of state-adding DBT, characterized by the $m$-uple $-M=(-1, \ldots,-m)$, which corresponds to $m$ successive reversed SUSY partnerships.

Lemma 4. If $\alpha_{-j} \in U, \forall j \leq m$, the final extension of a complete chain ofmstate-adding DBT applied to the TSIP is regular and satisfies the reverse shape invariance property

$$
V^{(-M)}(x ; \alpha)=V\left(x ; \alpha_{-m}\right)+E_{-m}(\alpha) .
$$

Its energy spectrum is given by $E_{j}^{(-M)}(\alpha)=E_{j-m}(\alpha), j \geq 0$, with the corresponding eigenstates

$$
\phi_{j}^{(-M)}(x ; \alpha)=\psi_{j-m}^{(-M)}(x ; \alpha) \sim \psi_{j}\left(x ; \alpha_{-m}\right) .
$$

Proof. From Eq. (81), an immediate recurrence gives

$$
V^{(-M)}(x ; \alpha)=V\left(x ; \alpha_{-m}\right)+\sum_{i=0}^{-m+1} E_{-1}\left(\alpha_{i}\right) .
$$


Equation (70) leading to

$$
E_{-m}(\alpha)=E_{-m+1}(\alpha)+E_{-1}\left(\alpha_{-m+1}\right)=\sum_{i=0}^{-m+1} E_{-1}\left(\alpha_{i}\right), \quad m \geq 1,
$$

we obtain

$$
V^{(-M)}(x ; \alpha)=V\left(x ; \alpha_{-m}\right)+E_{-m}(\alpha) .
$$

Due to the quasi-isospectrality property of the state-adding DBT, the Hamiltonian

$$
\widehat{H}^{(-M)}(\alpha)=-\frac{d^{2}}{d x^{2}}+V^{(-M)}(x ; \alpha)=\widehat{H}\left(\alpha_{-m}\right)+E_{-m}(\alpha)
$$

has the same energy spectrum as the initial potential with $m$ supplementary levels at the values $E_{-m}(\alpha)<\ldots<E_{-1}(\alpha)<0$. More precisely, the spectrum of $\widehat{H}^{(-M)}(\alpha)$ is given by

$$
\left\{\begin{array}{c}
E_{j}^{(-M)}(\alpha)=E_{-m+j}(\alpha) \\
\phi_{j}^{(-M)}(x ; \alpha)=\psi_{j-m}^{(-M)}(x ; \alpha)
\end{array}, \quad j \geq 0 .\right.
$$

The structural identity for the dispersion relation (70) gives

$$
E_{k}^{(-M)}(\alpha)=E_{k}\left(\alpha_{-m}\right)+E_{-m}(\alpha)
$$

which combined with Eqs. (96) and (95), implies

$$
\widehat{H}\left(\alpha_{-m}\right) \phi_{k}^{(-M)}(x ; \alpha)=E_{k}\left(\alpha_{-m}\right) \phi_{k}^{(-M)}(x ; \alpha) .
$$

This means in particular

$$
\phi_{k}^{(-M)}(x ; \alpha)=\psi_{k-m}^{(-M)}(x ; \alpha) \sim \psi_{k}\left(x ; \alpha_{-m}\right),
$$

when $\alpha_{-m} \in U$ (in which case $\psi_{k}\left(x ; \alpha_{-m}\right)$ satisfies the Dirichlet boundary conditions at the limit of the definition interval).

Equation (95) ensures the regularity of the potential $V^{(-M)}$ which, applying the Crum formula (16), can also be written as (see Eq. (25))

$$
V^{(-M)}(x ; \alpha)=V(x ; \alpha)-2\left(\log W^{(-M)}(x ; \alpha)\right)^{\prime \prime} .
$$

Consequently from Eqs. (95) and (101), we deduce the nodeless character of the Wronskian $W^{(-M)}(x)$ for every value of $m$ for which $\alpha_{-m} \in U$.

Comparing Eqs. (95) and (101) also gives the following identity for the eigenfunctions of the initial PTSIP:

Lemma 5. If $\alpha_{-m} \in U$

$$
\left\{\begin{array}{cl}
\psi_{k+m}\left(x ; \alpha_{-m}\right) \sim \frac{W^{(-M, k)}(x ; \alpha)}{W^{(-M)}(x ; \alpha)}=\frac{W\left(\psi_{-1}, \ldots, \psi_{-m}, \psi_{k} \mid x ; \alpha\right)}{W\left(\psi_{-1}, \ldots, \psi_{-m} \mid x ; \alpha\right)}, & k \geq 0, \\
\psi_{k+m}\left(x ; \alpha_{-m}\right) \sim \frac{W^{(-M /-k)}(x ; \alpha)}{W^{(-M)}(x ; \alpha)}=\frac{W\left(\psi_{-1}, \ldots, \psi_{-k+1}, \psi_{-k-1}, \ldots, \psi_{-m} \mid x ; \alpha\right)}{W\left(\psi_{-1}, \ldots, \psi_{-m} \mid x ; \alpha\right)}, \quad 0>k \geq-m .
\end{array}\right.
$$

Proof. The eigenstates of $V^{(-M)}$ associated to the positive levels $(k \geq 0)$ are given by (see Eq. (15))

$$
\psi_{k}^{(-M)}(x ; \alpha)=\frac{W^{(-M, k)}(x ; \alpha)}{W^{(-M)}(x ; \alpha)}, \quad k \geq 0,
$$

while the eigenstates of negative energies are given by (see Eq. (25))

$$
\psi_{-k}^{(-M)}(x ; \alpha)=\frac{W^{(-M /-k)}(x ; \alpha)}{W^{(-M)}(x ; \alpha)}, \quad k>0,
$$

all these eigenstates being regular on the definition domain.

The comparison of the expressions (100) and (103) allows to deduce directly Eq. (102). 


\section{B. Enlarged Krein-Adler theorem for TSIP and Wronskian identities for mixed extensions of TSIP}

By the above procedure, we have obtained a potential $V^{(-M)}$ whose spectrum is completely filled until the level $E_{-m}(\alpha)$. Up to a global translation and a shift in the parameters, it coincides with the initial potential. By considering $V^{(-M)}$ as an intermediate step, we can apply now an usual chain of state-deleting DBT in the frame of the Krein-Adler theorem. ${ }^{57,58}$ This suppresses some sequences in the spectrum and the global chain which is composed of both state-adding and state-deleting DBT in a mixed chain.

We then obtain the following generalization of the Krein-Adler theorem:

Enlarged Krein-Adler theorem for TSIP: Consider a TSIP $V(x ; \alpha), \alpha \in U$, with a discrete $\operatorname{spectrum}\left(E_{n}, \psi_{n}\right)_{n \in\left\{0, \ldots, n_{\max }\right\} \subseteq \mathbb{N}}$ and a mixed chain of state-adding and state-deleting DBT, characterized by them-uple $N_{m}=\left(n_{1}, \ldots, n_{m}\right)$ of positive (state-deleting DBT) and negative (state-deleting $D B T)$ integers. Let $n_{<}=\min _{1 \leq j \leq m}\left(n_{j}\right)$. When $\alpha_{j} \in U, \forall j \geq n_{<}$, the extended potential

$$
V^{\left(N_{m}\right)}(x ; \alpha)=V(x ; \alpha)+2\left(\ln \left|W\left(\psi_{n_{1}}, \ldots, \psi_{n_{m}} \mid x ; \alpha\right)\right|\right)^{\prime \prime}
$$

is regular iff its spectrum is a subset of $\left\{E_{j \in \mathbb{Z}}\right\}$ containing only even gaps (gaps constituted by an even number of consecutive missing levels).

Before proceeding to the proof, we introduce the following notation:

Let $\pm L$ be the $l$-uple associated to a complete chain of $l$ state-adding DBT $-L=(-1$, $\ldots,-l)$ or state-deleting DBT $L=(0,1, \ldots, l-1),\left\{l_{1}, \ldots, l_{k}\right\}$ a set of $k$ indices such that $\pm L \cap\left\{l_{1}, \ldots, l_{k}\right\}=\left\{l_{i_{1}}, \ldots, l_{i_{j}}\right\}$ and $\left\{l_{1}, \ldots, l_{k}\right\} /\left\{l_{i_{1}}, \ldots, l_{i_{j}}\right\}=\left\{l_{p_{1}}, \ldots, l_{p_{k-j}}\right\}, \quad j \leq l, k$. Then, $-L / / l_{1}, \ldots, l_{k}$ is the $(l+k-2 j)$-uple obtained by suppressing in $\pm L$ the indices $l_{i_{1}}, \ldots, l_{i_{j}}$ and adding to the resulting $(l-j)$-uple the indices $l_{p_{1}}, \ldots, l_{p_{k-j}}$

$$
\pm L / / l_{1}, \ldots, l_{k}=\left(\left( \pm L / l_{i_{1}}, \ldots, l_{i_{j}}\right), l_{p_{1}}, \ldots, l_{p_{k-j}}\right) .
$$

Proof. Let $-n_{<}=\min _{1 \leq j \leq m}\left(n_{j}\right)$ and $-N_{<}=\left(-1, \ldots,-n_{<}\right)$. We can always consider $N_{m}$ as constituted by the juxtaposition of the complete chain $-N_{<}$of $n_{<}$state-adding DBT and of a chain $\left(n_{j_{1}}, \ldots, n_{j_{k}}\right)$ of $k$ state-deleting DBT such that $N_{m}=-N_{<} / / n_{j_{1}}, \ldots, n_{j_{k}} . V^{\left(-N_{<}\right)}$being regular (see Lemma 4), the Krein-Adler theorem ensures that the extended potential generated by the chain of state deleting DBT based on the eigenfunctions $\psi_{n_{j_{l}}}^{\left(-N_{<}\right)}(x ; \alpha), \quad l=1, \ldots, k$, of $V^{\left(-N_{<}\right)}$is regular iff the chain $\left(n_{j_{1}}, \ldots, n_{j_{k}}\right)$ is of the Krein-Adler type. Using Lemma 2, the final extension $V^{\left(-N_{<}, n_{j_{1}}, \ldots, n_{j_{k}}\right)}$ can still be written

$$
V^{\left(-N_{<}, n_{j_{1}}, \ldots, n_{j_{k}}\right)}(x ; \alpha)=V^{\left(-N_{<} / / n_{j_{1}}, \ldots, n_{j_{k}}\right)}(x ; \alpha)=V^{\left(N_{m}\right)}(x ; \alpha),
$$

which is then regular iff its spectrum, that is, the set of integers $\left\{n \geq-n_{<}\right\} /\left\{n_{j_{1}}, \ldots, n_{j_{k}}\right\}$, contains only even gaps.

Combining Lemmas 2, 4, and 5, we then obtain the following result:

Theorem 1. Consider a mixed chain of DBT associated to $-M / / n_{1}, \ldots, n_{k}$ (where $m<0,-M$ $\left.=(-1, \ldots,-m),-m \leq \min _{i=1, \ldots, k}\left(n_{i}\right)\right)$. Suppose that $\alpha_{-j} \in U, \forall j \leq m$. Then, when this chain is applied to the TSIP $V(x ; \alpha)$, the final extension can be written as

$$
V^{\left(-M / / n_{1}, \ldots, n_{k}\right)}(x ; \alpha)=V^{\left(n_{1}+m, \ldots, n_{k}+m\right)}\left(x ; \alpha_{-m}\right)+E_{-m}(\alpha),
$$

and is regular iff $\left(n_{1}, \ldots, n_{k}\right)$ is of the Krein-Adler type or equivalently iff the spectrum of $V^{\left(-M / / n_{1}, \ldots, n_{k}\right)}$ contains only even gaps. 
The eigenstates of $V^{\left(-M / n_{1}, \ldots, n_{k}\right)}$ admit then the following Wronskian representation $\left(j \notin\left\{n_{1}\right.\right.$, $\left.\left.\ldots, n_{k}\right\}, \quad j \geq-m\right)$

$$
\begin{aligned}
\psi_{j}^{\left(-M / / n_{1}, \ldots, n_{k}\right)}(x ; \alpha) & =\frac{W^{\left(-M / / n_{1}, \ldots, n_{k}, j\right)}(x ; \alpha)}{W^{\left(-M / / n_{1}, \ldots, n_{k}\right)}(x ; \alpha)} \sim \frac{W^{\left(n_{1}+m, \ldots, n_{k}+m, j+m\right)}\left(x ; \alpha_{-m}\right)}{W^{\left(n_{1}+m, \ldots, n_{k}+m\right)}\left(x ; \alpha_{-m}\right)} \\
& =\psi_{j+m}^{\left(n_{1}+m, \ldots, n_{k}+m\right)}\left(x ; \alpha_{-m}\right) .
\end{aligned}
$$

Proof. Using Eqs. (93) and (100), we can write

$$
\begin{aligned}
V^{\left(-M / / n_{1}, \ldots, n_{k}\right)}(x ; \alpha) & =V^{\left(-M, n_{1}, \ldots, n_{k}\right)}(x ; \alpha) \\
& =V^{(-M)}(x ; \alpha)+2\left(\log \left(W\left(\psi_{n_{1}}^{(-M)}, \ldots, \psi_{n_{k}}^{(-M)} \mid x ; \alpha\right)\right)\right)^{\prime \prime} \\
& =V\left(x ; \alpha_{-m}\right)+E_{-m}(\alpha)+2\left(\log \left(W\left(\psi_{n_{1}+m}, \ldots, \psi_{n_{k}+m} \mid x ; \alpha_{-m}\right)\right)\right)^{\prime \prime} \\
& =V^{\left(n_{1}+m, \ldots, n_{k}+m\right)}\left(x ; \alpha_{-m}\right)+E_{-m}(\alpha)
\end{aligned}
$$

which implies

$$
V^{\left(-M / / n_{1}, \ldots, n_{k}\right)}(x ; \alpha)=V^{\left(n_{1}+m, \ldots, n_{k}+m\right)}\left(x ; \alpha_{-m}\right)+E_{-m}(\alpha) .
$$

The regularity condition results from the enlarged Krein-Adler theorem for TSIP.

Moreover, if $j \geq-m$

$$
\begin{aligned}
\psi_{j}^{\left(-M / / n_{1}, \ldots, n_{k}\right)}(x ; \alpha) & \sim \psi_{j}^{\left(-M, n_{1}, \ldots, n_{k}\right)}(x ; \alpha)=\frac{W\left(\psi_{n_{1}}^{(-M)}, \ldots, \psi_{n_{k}}^{(-M)}, \psi_{j}^{(-M)} \mid x ; \alpha\right)}{W\left(\psi_{n_{1}}^{(-M)}, \ldots, \psi_{n_{k}}^{(-M)} \mid x ; \alpha\right)} \\
& \sim \frac{W\left(\psi_{n_{1}+m}, \ldots, \psi_{n_{k}+m}, \psi_{j+m} \mid x ; \alpha_{-m}\right)}{W\left(\psi_{n_{1}+m}, \ldots, \psi_{n_{k}+m} \mid x ; \alpha_{-m}\right)} .
\end{aligned}
$$

If $\alpha_{-m} \in U$, in which case $\psi_{j}^{\left(n_{1}+m, \ldots, n_{k}+m\right)}\left(x ; \alpha_{-m}\right)$ satisfies the required Dirichlet boundary conditions, we then have

$$
\psi_{j}^{\left(-M / / n_{1}, \ldots, n_{k}\right)}(x ; \alpha) \sim \psi_{j+m}^{\left(n_{1}+m, \ldots, n_{k}+m\right)}\left(x ; \alpha_{-m}\right),
$$

that is,

$$
\frac{W^{\left(-M / / n_{1}, \ldots, n_{k}, j\right)}(x ; \alpha)}{W^{\left(-M / / n_{1}, \ldots, n_{k}\right)}(x ; \alpha)} \sim \frac{W^{\left(n_{1}+m, \ldots, n_{k}+m, j+m\right)}\left(x ; \alpha_{-m}\right)}{W^{\left(n_{1}+m, \ldots, n_{k}+m\right)}\left(x ; \alpha_{-m}\right)} .
$$

\section{EXAMPLES OF APPLICATION}

In what follows, we apply the general formalism developed above to some particular PTSIP. As an illustration, we consider the particular chain $-M / / n_{1}, \ldots, n_{k}=(-3,1,2)$ for $m=3$, i.e. $-M=(-1,-2,-3)$ and $k=4$ with $\left(n_{1}, \ldots, n_{4}\right)=(-2,-1,1,2)$. Equations (108) and (109) become in this case

$$
\left\{\begin{array}{c}
V^{(-3,1,2)}(x ; \alpha)=V^{(1,2,4,5)}\left(x ; \alpha_{-3}\right)+E_{-3}(\alpha) \\
W^{(-3,1,2, j)}(x ; \alpha) / W^{(-3,1,2)}(x ; \alpha) \sim W^{(1,2,4,5, j+3)}\left(x ; \alpha_{-3}\right) / W^{(1,2,4,5)}\left(x ; \alpha_{-3}\right) .
\end{array}\right.
$$

\section{A. The harmonic oscillator}

The harmonic oscillator potential is defined on the real line by

$$
V(x ; \omega)=\frac{\omega^{2}}{4} x^{2}-\frac{\omega}{2}, \omega \in \mathbb{R} .
$$


With Dirichlet boundary conditions at infinity and supposing $\omega \in \mathbb{R}^{+}, V(x ; \omega)$ has the following $\operatorname{spectrum}\left(z=\omega x^{2} / 2\right)$ :

$$
\left\{\begin{array}{c}
E_{n}(\omega)=n \omega \\
\psi_{n}(x ; \omega)=H_{n}(\sqrt{\omega / 2} x) \exp \left(-\omega x^{2} / 4\right)=H_{n}(\sqrt{z}) \exp (-z / 2)
\end{array}, \quad n \geq 0 .\right.
$$

It is the most simple example of TSIP, with $\alpha=\omega \in \mathbb{R}$ and $\varepsilon=0$ (the parameter translation is of zero amplitude $\alpha_{1}=\alpha=\omega$ ), that is,

$$
V^{(0)}(x ; \omega)=V(x ; \omega)+\omega .
$$

The $\Gamma_{3}$ symmetry acts as ${ }^{32}$

$$
\omega \stackrel{\Gamma_{3}}{\rightarrow}(-\omega),\left\{\begin{array}{c}
V(x ; \omega) \stackrel{\Gamma_{3}}{\rightarrow} V(x ;-\omega)=V(x ; \omega)+\omega, \\
\psi_{n}(x ; \omega) \stackrel{\Gamma_{3}}{\rightarrow} \psi_{n}(x ;-\omega)=\psi_{-(n+1)}(x ; \omega),
\end{array}\right.
$$

and then generates the following quasi-polynomial eigenfunctions of the disconjugacy sector:

$$
\left\{\begin{array}{c}
E_{-n}(\omega)=-n \omega \\
\psi_{-n}(x ; \omega)=H_{n-1}(i \sqrt{\omega / 2} x) \exp \left(\omega x^{2} / 4\right)=\psi_{n-1}(x ;-\omega)
\end{array}, \quad n \geq 1,\right.
$$

diverging at both infinities with a parity equal to the one of $n-1$. The disconjugacy theorem implies immediately that $\psi_{-n}(x ; \omega)$ is free of node for $n$ odd. Then $1 / \psi_{-n}$ is regular on the real line and we can verify it is also normalizable. The DBT $A\left(w_{-n}(\omega)\right), n$ odd, is consequently state-adding. Note that, for every odd $n$, the regular one-step extended potential $V^{(-n)}(x ; \omega)$ presents an even gap in its spectrum (the levels from -1 to $-n+1$ are missing), in agreement with the enlarged Krein-Adler theorem.

From Theorem 1, we deduce

$$
V^{\left(-M / / n_{1}, \ldots, n_{k}\right)}(x ; \omega)=V(x ; \omega)+2\left(\log \left(W^{\left(-M / / n_{1}, \ldots, n_{k}\right)}(x ; \omega)\right)\right)^{\prime \prime}=V^{\left(n_{1}+m, \ldots, n_{k}+m\right)}(x ; \omega)-m \omega,
$$

without any limitation on the length of the complete chain $-M$. Up to an additive constant, the final potential coincides then exactly with the one obtained by applying the chain of state-deleting DBT $\left(n_{1}, \ldots, n_{k}\right)$ to the initial harmonic potential $V(x ; \omega)$. Moreover,

$$
\begin{aligned}
\psi_{j}^{\left(-M / / n_{1}, \ldots, n_{k}\right)}(x ; \omega) & =\frac{W^{\left(-M / / n_{1}, \ldots, n_{k}, j\right)}(x ; \omega)}{W^{\left(-M / / n_{1}, \ldots, n_{k}\right)}(x ; \omega)} \sim \frac{W^{\left(n_{1}+m, \ldots, n_{k}+m, j+m\right)}(x ; \omega)}{W^{\left(n_{1}+m, \ldots, n_{k}+m\right)}(x ; \omega)} \\
& =\psi_{j+m}^{\left(n_{1}+m, \ldots, n_{k}+m\right)}(x ; \omega), \quad j \geq-m .
\end{aligned}
$$

For the considered example (see Eq. (115)), we obtain

$$
V^{(-3,1,2)}(x ; \omega)=V^{(1,2,4,5)}(x ; \omega)-3 \omega
$$

and

$$
\frac{W\left(\psi_{-3}, \psi_{1}, \psi_{2}, \psi_{j} \mid x ; \omega\right)}{W\left(\psi_{-3}, \psi_{1}, \psi_{2} \mid x ; \omega\right)} \sim \frac{W\left(\psi_{1}, \psi_{2}, \psi_{4}, \psi_{5}, \psi_{j+3} \mid x ; \omega\right)}{W\left(\psi_{1}, \psi_{2}, \psi_{4}, \psi_{5} \mid x ; \omega\right)}
$$

Using Eqs. (117) and (120) and usual properties of Wronskians, ${ }^{55}$ this leads to $(\omega=2)$

$$
\begin{aligned}
& \frac{W\left(H_{2}(i x) \exp \left(x^{2}\right), H_{1}(x), H_{2}(x), H_{j}(x) \mid x\right)}{W\left(H_{2}(i x) \exp \left(x^{2}\right), H_{1}(x), H_{2}(x) \mid x\right)} \\
& \sim \frac{W\left(H_{1}(x), H_{2}(x), H_{4}(x), H_{5}(x), H_{j+3}(x) \mid x\right)}{W\left(H_{1}(x), H_{2}(x), H_{4}(x), H_{5}(x) \mid x\right)} .
\end{aligned}
$$

From the Rodrigues formula, ${ }^{47,48}$ it results

$$
\frac{d^{k}}{d x^{k}}\left(\exp \left(x^{2}\right) H_{n}(i x)\right)=\frac{1}{i^{k}} \exp \left(x^{2}\right) H_{n+k}(),
$$


and since

$$
\frac{d^{k}}{d x^{k}}\left(H_{n}(x)\right)=2^{k} \frac{n !}{(n-k) !} H_{n-k}(x),
$$

we obtain the following bilinear determinantal identity for Hermite polynomials:

$$
\begin{gathered}
\left|\begin{array}{cccc}
H_{2}(i x) & H_{1}(x) & H_{2}(x) & H_{j}(x) \\
\frac{1}{i} H_{3}(i x) & 2 H_{0}(x) & 4 H_{1}(x) & 2 j H_{j-1}(x) \\
-H_{4}(i x) & 0 & 8 H_{0}(x) & 4(j-1)_{2} H_{j-2}(x) \\
-\frac{1}{i} H_{5}(i x) & 0 & 0 & 8(j-2)_{3} H_{j-3}(x)
\end{array}\right| \\
\left|\begin{array}{cccc}
H_{2}(i x) & H_{1}(x) & H_{2}(x) \\
\frac{1}{i} H_{3}(i x) & 2 H_{0}(x) & 4 H_{1}(x) \\
-H_{4}(i x) & 0 & 8 H_{0}(x)
\end{array}\right| \\
\sim \begin{array}{ccccc}
H_{1}(x) & H_{2}(x) & H_{4}(x) & H_{5}(x) & H_{j+3}(x) \\
H_{0}(x) & 2 H_{1}(x) & 4 H_{3}(x) & 5 H_{4}(x) & (j+3) H_{j+2}(x) \\
0 & 2 H_{0}(x) & 12 H_{2}(x) & 20 H_{3}(x) & (j+2)_{2} H_{j+1}(x) \\
0 & 0 & 24 H_{1}(x) & 60 H_{2}(x) & (j+1)_{3} H_{j}(x) \\
0 & 0 & 24 H_{0}(x) & 120 H_{1}(x) & (j)_{4} H_{j-1}(x)
\end{array} \mid
\end{gathered}
$$

where $(x)_{n}$ is the usual Pochammer symbol: $(x)_{n}=x(x+1) \ldots(x+n-1){ }^{48}$

\section{B. The isotonic oscillator}

The isotonic oscillator potential (with zero ground level $E_{0}=0$ )) is defined on the positive half line $] 0,+\infty[$ by

$$
V(x ; \omega, \alpha)=\frac{\omega^{2}}{4} x^{2}+\frac{(\alpha+1 / 2)(\alpha-1 / 2)}{x^{2}}-\omega(\alpha+1), \quad|\alpha|>1 / 2 .
$$

If we add Dirichlet boundary conditions at 0 and infinity and if we suppose $\alpha>1 / 2$ and $\alpha \notin \mathbb{N}$, it has the following spectrum $\left(z=\omega x^{2} / 2\right)$ :

$$
\left\{\begin{array}{c}
E_{n}(\omega)=2 n \omega \\
\psi_{n}(x ; \omega, \alpha)=x^{\alpha+1 / 2} e^{-z / 2} L_{n}^{\alpha}(z)
\end{array}, \quad n \geq 0 .\right.
$$

It is a TSIP, with $(\omega, \alpha) \in \mathbb{R}^{2}$ and $\varepsilon=(0,+1)$

$$
V^{(0)}(x ; \omega, \alpha)=V\left(x ; \omega, \alpha_{1}\right)+2 \omega .
$$

It possesses a $\Gamma_{3}$ symmetry which acts as ${ }^{32}$

$$
(\omega, \alpha) \stackrel{\Gamma_{3}}{\rightarrow}(-\omega,-\alpha),\left\{\begin{array}{c}
V(x ; \omega, \alpha) \stackrel{\Gamma_{3}}{\rightarrow} V(x ;-\omega,-\alpha)=V(x ; \omega, \alpha)+\omega, \\
\psi_{n}(x ; \omega, \alpha) \stackrel{\Gamma_{3}}{\rightarrow} \psi_{n}(x ;-\omega,-\alpha)=\psi_{-(n+1)}(x ; \omega, \alpha),
\end{array}\right.
$$


and then generates the following quasi-polynomial eigenfunctions of the disconjugacy sector:

$$
\left\{\begin{array}{c}
E_{-n}(\omega)=-2 n \omega \\
\psi_{-n}(x ; \omega, \alpha)=x^{-\alpha+1 / 2} e^{z / 2} L_{n-1}^{-\alpha}(-z)
\end{array}, \quad n \geq 1 .\right.
$$

Since $^{47,48}$

$$
\left\{\begin{array}{c}
L_{n}^{-\alpha}(-x) \underset{x \rightarrow 0^{+}}{\rightarrow} \frac{(-\alpha+1)_{n}}{n !}=\frac{(-\alpha+1 \ldots(-\alpha+n)}{n !} \\
L_{n}^{-\alpha}(-x) \underset{x \rightarrow+\infty}{\sim} \frac{1}{n !} x^{n}
\end{array}\right.
$$

$\psi_{-n}$ diverges at 0 and infinity $(\alpha>1 / 2)$. Moreover, when $n<\alpha+1, \psi_{-n}$ has an asymptotic sign equal to $(-1)^{n-1}$ and when $n>1+[\alpha]([\alpha]$ being the integer part of $\alpha), \psi_{-n}$ has an asymptotic sign equal to $(-1)^{[\alpha]}$, independently of $n$.

Note that the disconjugacy theorem implies immediately that for $n<\alpha+1, \psi_{-n}(x ; \omega, \alpha)$ is free of node when $n$ is odd and that for $n>1+[\alpha], \psi_{-n}(x ; \omega, \alpha)$ is free of node when $[\alpha]$ is even. Then $1 / \psi_{-n}$ is regular on the real line and it is also normalizable (see Eq. (133)). This is in agreement with the classical Kienast-Lawton-Hahn theorem on the number of negative zeros of the Laguerre polynomials. $^{32,47,48}$ Consequently, in these cases, the DBT $A\left(w_{-n}(\omega, \alpha)\right)$ is state-adding.

When $n<\alpha+1$ and $n$ odd, the regular one-step extended potential $V^{(-n)}(x ; \omega, \alpha)$ presents an even gap in its spectrum (the levels from -1 to $-n+1$ are missing), in agreement with the enlarged Krein-Adler theorem.

However, when $n>1+[\alpha]$ and $[\alpha]$ even, $V^{(-n)}(x ; \omega, \alpha)$ is regular for any parity of $n$ and consequently its spectrum can present odd gaps (when $n$ is even, the $n-1$ levels $-n-1, \ldots,-1$ are missing). Nevertheless, for $m>1+[\alpha]$ we satisfy no longer the condition $\left|\alpha_{-j}\right|<1 / 2, \forall j \leq m$. Indeed, $\left|\alpha_{-[\alpha]}\right|>1 / 2$ or $\left|\alpha_{-([\alpha]+1)}\right|>1 / 2$ and the potentials $V\left(x ; \omega, \alpha_{-[\alpha]}\right)$ or $V\left(x ; \omega, \alpha_{-([\alpha]+1)}\right)$ have a singular behaviour.

To stay in the conditions of applicability of the enlarged Krein-Adler theorem and of the Theorem 1, we have then to consider only seed functions satisfying $n<\alpha+1$.

If $\alpha>m+1 / 2$, we have (see Theorem 1)

$$
V^{\left(-M / / n_{1}, \ldots, n_{k}\right)}(x ; \omega, \alpha)=V^{\left(n_{1}+m, \ldots, n_{k}+m\right)}\left(x ; \omega, \alpha_{-m}\right)-2 m \omega .
$$

The eigenstates of this extended potential satisfy for $j \geq-m$

$$
\begin{aligned}
\psi_{j}^{\left(-M / / n_{1}, \ldots, n_{k}\right)}(x ; \omega, \alpha) & =\frac{W^{\left(-M / / n_{1}, \ldots, n_{k}, j\right)}(x ; \omega, \alpha)}{W^{\left(-M / / n_{1}, \ldots, n_{k}\right)}(x ; \omega, \alpha)} \sim \frac{W^{\left(n_{1}+m, \ldots, n_{k}+m, j+m\right)}\left(x ; \omega, \alpha_{-m}\right)}{W^{\left(n_{1}+m, \ldots, n_{k}+m\right)}\left(x ; \omega, \alpha_{-m}\right)} \\
& =\psi_{j+m}^{\left(n_{1}+m, \ldots, n_{k}+m\right)}\left(x ; \omega, \alpha_{-m}\right) .
\end{aligned}
$$

In the considered example we obtain, for $\alpha>7 / 2$

$$
V^{(-3,1,2)}(x ; \omega, \alpha)=V^{(1,2,4,5)}\left(x ; \omega, \alpha_{-3}\right)-3 \omega
$$

and

$$
\frac{W\left(\psi_{-3}, \psi_{1}, \psi_{2}, \psi_{j} \mid x ; \omega, \alpha\right)}{W\left(\psi_{-3}, \psi_{1}, \psi_{2} \mid x ; \omega, \alpha\right)} \sim \frac{W\left(\psi_{1}, \psi_{2}, \psi_{4}, \psi_{5}, \psi_{j+3} \mid x ; \omega, \alpha_{-3}\right)}{W\left(\psi_{1}, \psi_{2}, \psi_{4}, \psi_{5} \mid x ; \omega, \alpha_{-3}\right)} .
$$

Using Eqs. (130) and (133) and the usual properties of Wronskians, ${ }^{55}$ this leads to

$$
\begin{aligned}
& \frac{W\left(z^{-\alpha} e^{z} L_{2}^{-\alpha}(-z), L_{1}^{\alpha}(z), L_{2}^{\alpha}(z), L_{j}^{\alpha}(z) \mid x\right)}{W\left(z^{-\alpha} e^{z} L_{2}^{-\alpha}(-z), L_{1}^{\alpha}(z), L_{2}^{\alpha}(z) \mid x\right)} \\
& \sim \frac{W\left(L_{1}^{\alpha_{-3}}(z), L_{2}^{\alpha_{-3}}(z), L_{4}^{\alpha_{-3}}(z), L_{5}^{\alpha_{-3}}(z), L_{j+3}^{\alpha_{-3}}(z) \mid x\right)}{W\left(L_{1}^{\alpha_{-3}}(z), L_{2}^{\alpha_{-3}}(z), L_{4}^{\alpha_{-3}}(z), L_{5}^{\alpha_{-3}}(z) \mid x\right)},
\end{aligned}
$$


or, with $W\left(y_{1}, \ldots, y_{m} \mid x\right)=\left(\frac{d z}{d x}\right)^{m(m-1) / 2} W\left(y_{1}, \ldots, y_{m} \mid z\right)^{55}$

$$
\begin{aligned}
& z \frac{W\left(z^{-\alpha} e^{z} L_{2}^{-\alpha}(-z), L_{1}^{\alpha}(z), L_{2}^{\alpha}(z), L_{j}^{\alpha}(z) \mid z\right)}{W\left(z^{-\alpha} e^{z} L_{2}^{-\alpha}(-z), L_{1}^{\alpha}(z), L_{2}^{\alpha}(z) \mid z\right)} \\
& \quad \sim \frac{W\left(L_{1}^{\alpha_{-3}}(z), L_{2}^{\alpha_{-3}}(z), L_{4}^{\alpha_{-3}}(z), L_{5}^{\alpha_{-3}}(z), L_{j+3}^{\alpha_{-3}}(z) \mid z\right)}{W\left(L_{1}^{\alpha_{-3}}(z), L_{2}^{\alpha_{-3}}(z), L_{4}^{\alpha_{-3}}(z), L_{5}^{\alpha_{-3}}(z) \mid z\right)} .
\end{aligned}
$$

We have $\mathrm{e}^{47,48}$

$$
\frac{d^{k}}{d z^{k}}\left(L_{n}^{\alpha}(z)\right)=(-1)^{k} L_{n-k}^{\alpha+k}(z)
$$

and from the Rodrigues formula for Laguerre polynomials it results

$$
\frac{d^{k}}{d z^{k}}\left(z^{-\alpha} e^{z} L_{n}^{-\alpha}(-z)\right)=\frac{(n+k) !}{n !} z^{-\alpha-k} e^{z} L_{n+k}^{-\alpha-k}(-z) .
$$

Equation (140) then gives the following bilinear determinantal identity for Laguerre polynomials:

$$
\begin{aligned}
& z \frac{\left|\begin{array}{cccc}
L_{2}^{-\alpha}(-z) & L_{1}^{\alpha}(z) & L_{2}^{\alpha}(z) & L_{j}^{\alpha}(z) \\
-3 z^{-1} L_{3}^{-\alpha-1}(-z) & L_{0}^{\alpha+1}(z) & L_{1}^{\alpha+1}(z) & L_{j-1}^{\alpha+1}(z) \\
12 z^{-2} L_{4}^{-\alpha-2}(-z) & 0 & L_{0}^{\alpha+2}(z) & L_{j-2}^{\alpha+2}(z) \\
-60 z^{-3} L_{5}^{-\alpha-3}(-z) & 0 & 0 & L_{j-3}^{\alpha+3}(z)
\end{array}\right|}{\left|\begin{array}{ccc}
L_{2}^{-\alpha}(-z) & L_{1}^{\alpha}(z) & L_{2}^{\alpha}(z) \\
-3 z^{-1} L_{3}^{-\alpha-1}(-z) & L_{0}^{\alpha+1}(z) & L_{1}^{\alpha+1}(z) \\
12 z^{-2} L_{4}^{-\alpha-2}(-z) & 0 & L_{0}^{\alpha+2}(z)
\end{array}\right|} \\
& \sim \frac{\left|\begin{array}{ccccc}
L_{1}^{\alpha-3}(z) & L_{2}^{\alpha-3}(z) & L_{4}^{\alpha-3}(z) & L_{5}^{\alpha-3}(z) & L_{j+3}^{\alpha-3}(z) \\
L_{0}^{\alpha-2}(z) & L_{1}^{\alpha-2}(z) & L_{3}^{\alpha-2}(z) & L_{4}^{\alpha-2}(z) & L_{j+2}^{\alpha-2}(z) \\
0 & L_{0}^{\alpha-1}(z) & L_{2}^{\alpha-1}(z) & L_{3}^{\alpha-1}(z) & L_{j+1}^{\alpha-1}(z) \\
0 & 0 & L_{1}^{\alpha}(z) & L_{2}^{\alpha}(z) & L_{j}^{\alpha}(z) \\
0 & 0 & L_{0}^{\alpha+1}(z) & L_{1}^{\alpha+1}(z) & L_{j-1}^{\alpha+1}(z)
\end{array}\right|}{\left|\begin{array}{cccc}
L_{1}^{\alpha-3}(z) & L_{2}^{\alpha-3}(z) & L_{4}^{\alpha-3}(z) & L_{5}^{\alpha-3}(z) \\
L_{0}^{\alpha-2}(z) & L_{1}^{\alpha-2}(z) & L_{3}^{\alpha-2}(z) & L_{4}^{\alpha-2}(z) \\
0 & L_{0}^{\alpha-1}(z) & L_{2}^{\alpha-1}(z) & L_{3}^{\alpha-1}(z) \\
0 & 0 & L_{1}^{\alpha}(z) & L_{2}^{\alpha}(z)
\end{array}\right|} .
\end{aligned}
$$

\section{The TDPT potential}

The TDPT potential is defined on the interval $] 0, \pi / 2[$ by

$$
V(x ; \alpha, \beta)=\frac{(\alpha+1 / 2)(\alpha-1 / 2)}{\sin ^{2} x}+\frac{(\beta+1 / 2)(\beta-1 / 2)}{\cos ^{2} x}-(\alpha+\beta+1)^{2},|\alpha|,|\beta|>1 / 2 .
$$


With Dirichlet boundary conditions at 0 and $\pi / 2$, it has, in the case $\alpha, \beta>1 / 2$, the following spectrum:

$$
\left\{\begin{array}{c}
E_{n}(\alpha, \beta)=\left(\alpha_{n}+\beta_{n}+1\right)^{2}-(\alpha+\beta+1)^{2}=4 n(\alpha+\beta+1+n) \\
\psi_{n}(x ; \alpha, \beta)=(\sin x)^{\alpha+1 / 2}(\cos x)^{\beta+1 / 2} P_{n}^{(\alpha, \beta)}(\cos 2 x)
\end{array}, n \in \mathbb{N},\right.
$$

where $P_{n}^{(\alpha, \beta)}$ are the usual Jacobi polynomials ${ }^{47,48}$ and where $\left(\alpha_{n}, \beta_{n}\right)=(\alpha+n, \beta+n)$.

It is a TSIP, with $(\alpha, \beta) \in \mathbb{R}^{2}$ and $\varepsilon=(+1,+1)$

$$
V^{(0)}(x ; \alpha, \beta)=V\left(x ; \alpha_{1}, \beta_{1}\right)+4(\alpha+\beta+2) .
$$

For this potential, the $\Gamma_{3}$ symmetry acts as

$$
(\alpha, \beta) \stackrel{\Gamma_{3}}{\rightarrow}(-\alpha,-\beta),\left\{\begin{array}{c}
V(x ; \alpha, \beta) \stackrel{\Gamma_{3}}{\rightarrow} V(x ;-\alpha,-\beta)=V(x ; \alpha, \beta)+4 \alpha(\beta+1) \\
\psi_{n}(x ; \alpha, \beta) \stackrel{\Gamma_{3}}{\rightarrow} \psi_{n}(x ;-\alpha,-\beta),
\end{array}\right.
$$

where $\psi_{n}(x ;-\alpha,-\beta)$ satisfies

$$
\widehat{H}(-\alpha,-\beta) \psi_{n}(x ;-\alpha,-\beta)=E_{n}(-\alpha,-\beta) \psi_{n}(x ;-\alpha,-\beta),
$$

that is, using Eq. (147)

$$
\begin{aligned}
\widehat{H}(\alpha, \beta) \psi_{n}(x ;-\alpha,-\beta) & =(4 n(1+n-\alpha-\beta)-4 \alpha(\beta+1)) \psi_{n}(x ;-\alpha,-\beta) \\
& =E_{-(n+1)}(\alpha, \beta) \psi_{n}(x ;-\alpha,-\beta),
\end{aligned}
$$

from which we deduce $\mathcal{E}_{n, 3}(\alpha, \beta)=E_{-(n+1)}(\alpha, \beta)$ and

$$
\psi_{-(n+1)}(x ; \alpha, \beta)=\psi_{n}(x ;-\alpha,-\beta) .
$$

Then $\Gamma_{3}$ generates the following quasi-polynomial eigenfunctions of $V(x ; \alpha, \beta)$ :

$$
\left\{\begin{array}{c}
E_{-n}(\alpha, \beta)=4 n(n-1-\alpha-\beta), \\
\psi_{-n}(x ; \omega, \alpha)=(\sin x)^{-\alpha+1 / 2}(\cos x)^{-\beta+1 / 2} P_{n-1}^{(-\alpha,-\beta)}(\cos 2 x) .
\end{array}\right.
$$

In this case, they are only a finite number of them (satisfying $n<1+\alpha+\beta$ in which case $\left.E_{-n}(\alpha, \beta)<0\right)$ which are in the disconjugacy sector. Note that the quadratic dispersion relation $\left(E_{n}(\alpha, \beta)\right.$ as a function of $\left.n\right)$ is symmetric with respect to the value $-\frac{1+\alpha+\beta}{2}$ implying that the values of $-n$ we have to take into account are in fact limited to the set $\{-[(1+\alpha+\beta) / 2], \ldots,-1\}$ ( $[\lambda]$ being the integer part of $\lambda$ ). This corresponds to the fact that ${ }^{47,48}$

$$
P_{n}^{(-\alpha,-\beta)}(z) \sim F\left(-n, n+1+\alpha+\beta, \alpha+1, \frac{1-x}{2}\right),
$$

the right hand member being invariant under the transformation $n \rightarrow-(n+1+\alpha+\beta)$, i.e., the symmetry of $n$ with respect to $-\frac{1+\alpha+\beta}{2}$.

$\psi_{-n}$ diverges at 0 and infinity $(\alpha, \beta>1 / 2)$. Moreover, ${ }^{47,48}$

$$
\left\{\begin{array}{c}
P_{n}^{(-\alpha,-\beta)}(z) \underset{z \rightarrow 1^{-}}{\rightarrow} \frac{(-\alpha+1)_{n}}{n !}=\frac{(-\alpha+1) \ldots(-\alpha+n)}{n !}, \\
P_{n}^{(-\alpha,-\beta)}(z) \underset{z \rightarrow-1+}{\rightarrow}(-1)^{n} \frac{(-\beta+1)_{n}}{n !}=\frac{(\beta-1) \ldots(\beta-n)}{n !} .
\end{array}\right.
$$

When $(-\alpha+1)_{n-1}$ and $(-1)^{n-1}(-\beta+1)_{n-1}$ have the same sign or have opposite signs, $\psi_{-n}$ has an even or odd asymptotic parity. If we restrict ourselves to $n<\alpha+1$ and $n<\beta$ +1 , in which case we also have immediately $n<(1+\alpha+\beta) / 2$, the disconjugacy theorem then implies that $\psi_{-n}$ is free of node when $n$ is odd. Moreover, this is the condition to satisfy in order that $\left|\alpha_{-j}\right|,\left|\beta_{-j}\right|<1 / 2, \forall j \leq n$, which, as in the example of the isotonic oscillator, is a necessary condition for the use of the enlarged Krein-Adler theorem and of the theorem 1, to chains of DBT including seed functions until $\psi_{-n}$. We have then to consider only seed functions satisfying $n<\min (\alpha+1, \beta+1)$. 
Note that the disconjugacy theorem implies immediately that in this case $\psi_{-n}(x ; \alpha, \beta)$ is free of node for $n$ odd. Then $1 / \psi_{-n}$ is regular on the real line, normalizable (see Eq. (133)) and the DBT $A\left(w_{-n}(\alpha, \beta)\right)$ is state-adding.

From Theorem 1 , if $\alpha, \beta>m+1 / 2$, we then obtain a extended potential of the form

$$
V^{\left(-M / / n_{1}, \ldots, n_{k}\right)}(x ; \alpha, \beta)=V^{\left(n_{1}+m, \ldots, n_{k}+m\right)}\left(x ; \alpha_{-m}, \beta_{-m}\right)-4 m(1+\alpha+\beta-m),
$$

the eigenstates of which satisfy, for $j \geq-m$

$$
\begin{aligned}
\psi_{j}^{\left(-M / / n_{1}, \ldots, n_{k}\right)}(x ; \alpha, \beta) & =\frac{W^{\left(-M / / n_{1}, \ldots, n_{k}, j\right)}(x ; \alpha, \beta)}{W^{\left(-M / / n_{1}, \ldots, n_{k}\right)}(x ; \alpha, \beta)} \sim \frac{W^{\left(n_{1}+m, \ldots, n_{k}+m, j+m\right)}\left(x ; \alpha_{-m}, \beta_{-m}\right)}{W^{\left(n_{1}+m, \ldots, n_{k}+m\right)}\left(x ; \alpha_{-m}, \beta_{-m}\right)} \\
& =\psi_{j+m}^{\left(n_{1}+m, \ldots, n_{k}+m\right)}\left(x ; \alpha_{-m}, \beta_{-m}\right) .
\end{aligned}
$$

In the example considered above (see Eq. (115)), we obtain for $\alpha, \beta>7 / 2$

$$
V^{(-3,1,2)}(x ; \alpha, \beta)=V^{(1,2,4,5)}(x ; \alpha, \beta)-12(\alpha+\beta-2)
$$

and

$$
\frac{W\left(\psi_{-3}, \psi_{1}, \psi_{2}, \psi_{j} \mid x ; \alpha, \beta\right)}{W\left(\psi_{-3}, \psi_{1}, \psi_{2} \mid x ; \alpha, \beta\right)} \sim \frac{W\left(\psi_{1}, \psi_{2}, \psi_{4}, \psi_{5}, \psi_{j+3} \mid x ; \alpha_{-3}, \beta_{-3}\right)}{W\left(\psi_{1}, \psi_{2}, \psi_{4}, \psi_{5} \mid x ; \alpha_{-3}, \beta_{-3}\right)} .
$$

Using Eqs. (145) and (151) and usual properties of Wronskians, ${ }^{55}$ this leads to $(z=\cos 2 x)$

$$
\begin{gathered}
\frac{W\left((\sin x)^{-2 \alpha}(\cos x)^{-2 \beta} P_{2}^{(-\alpha,-\beta)}(z), P_{1}^{(\alpha, \beta)}(z), P_{2}^{(\alpha, \beta)}(z), P_{j}^{(\alpha, \beta)}(z) \mid x\right)}{W\left((\sin x)^{-2 \alpha}(\cos x)^{-2 \beta} P_{2}^{(-\alpha,-\beta)}(z), P_{1}^{(\alpha, \beta)}(z), P_{2}^{(\alpha, \beta)}(z) \mid x\right)} \\
\sim(\sin 2 x)^{-3} \frac{W\left(P_{1}^{\left(\alpha_{-3}, \beta_{-3}\right)}(z), P_{1}^{\left(\alpha_{-3}, \beta_{-3}\right)}(z), P_{1}^{\left(\alpha_{-3}, \beta_{-3}\right)}(z), P_{5}^{\left(\alpha_{-3}, \beta_{-3}\right)}(z), P_{j+3}^{\left(\alpha_{-3}, \beta_{-3}\right)}(z) \mid x\right)}{W\left(P_{1}^{\left(\alpha_{-3}, \beta_{-3}\right)}(z), P_{1}^{\left(\alpha_{-3}, \beta_{-3}\right)}(z), P_{1}^{\left(\alpha_{-3}, \beta_{-3}\right)}(z), P_{5}^{\left(\alpha_{-3}, \beta_{-3}\right)}(z) \mid x\right)},
\end{gathered}
$$

or, with $W\left(y_{1}, \ldots, y_{m} \mid x\right)=\left(\frac{d z}{d x}\right)^{m(m-1) / 2} W\left(y_{1}, \ldots, y_{m} \mid z\right)^{55}$

$$
\begin{aligned}
& \left(1-z^{2}\right) \frac{W\left((1-z)^{-\alpha}(1+z)^{-\beta} P_{2}^{(-\alpha,-\beta)}(z), P_{1}^{(\alpha, \beta)}(z), P_{2}^{(\alpha, \beta)}(z), P_{j}^{(\alpha, \beta)}(z) \mid z\right)}{W\left((1-z)^{-\alpha}(1+z)^{-\beta} P_{2}^{(-\alpha,-\beta)}(z), P_{1}^{(\alpha, \beta)}(z), P_{2}^{(\alpha, \beta)}(z) \mid z\right)} \\
\sim & \frac{W\left(P_{1}^{\left(\alpha_{-3}, \beta_{-3}\right)}(z), P_{2}^{\left(\alpha_{-3}, \beta_{-3}\right)}(z), P_{4}^{\left(\alpha_{-3}, \beta_{-3}\right)}(z), P_{5}^{\left(\alpha_{-3}, \beta_{-3}\right)}(z), P_{j+3}^{\left(\alpha_{-3}, \beta-3\right)}(z) \mid z\right)}{W\left(P_{1}^{\left(\alpha_{-3}, \beta_{-3}\right)}(z), P_{2}^{\left(\alpha_{-3}, \beta_{-3}\right)}(z), P_{4}^{\left(\alpha_{-3}, \beta_{-3}\right)}(z), P_{5}^{\left(\alpha_{-3}, \beta_{-3}\right)}(z) \mid z\right)} .
\end{aligned}
$$

We have ${ }^{47,48}$

$$
\frac{d^{k}}{d z^{k}}\left(P_{n}^{(\alpha, \beta)}(z)\right)=\frac{(1+\alpha+\beta+n)_{k}}{2^{k}} P_{n-k}^{\left(\alpha_{k}, \beta_{k}\right)}(z)
$$

and from the Rodrigues formula for the Jacobi polynomials, ${ }^{47,48}$ it results

$$
\frac{d^{k}}{d z^{k}}\left((1-z)^{-\alpha}(1+z)^{-\beta} P_{n}^{(-\alpha,-\beta)}(z)\right)=(-2)^{k} \frac{(n+k) !}{n !}(1-z)^{-\alpha_{k}}(1+z)^{-\beta_{k}} P_{n+k}^{\left(-\alpha_{k},-\beta_{k}\right)}(z) \text {. }
$$

Equation (159) then gives the following bilinear determinantal identity for Jacobi polynomials:

$$
\frac{\Pi_{-3,1,2, j}(z)}{\Pi_{-3,1,2}(z)} \sim \frac{\Pi_{1,2,4,5, j+3}(z)}{\Pi_{1,2,4,5}(z)},
$$


where

$$
\begin{aligned}
& \Pi_{-3,1,2, j}(z)=\left|\begin{array}{cccc}
\left(1-z^{2}\right)^{3} P_{2}^{(-\alpha,-\beta)}(z) & P_{1}^{(\alpha, \beta)}(z) & P_{2}^{(\alpha, \beta)}(z) & P_{j}^{(\alpha, \beta)}(z) \\
(-2)(n+1)\left(1-z^{2}\right)^{2} P_{3}^{\left(-\alpha_{1},-\beta_{1}\right)}(z) & \frac{(2+\alpha+\beta)_{1}}{2} P_{0}^{\left(\alpha_{1}, \beta_{1}\right)}(z) & \frac{(3+\alpha+\beta)_{1}}{2} P_{1}^{\left(\alpha_{1}, \beta_{1}\right)}(z) & \frac{(1+\alpha+\beta+j)_{1}}{2} P_{j-1}^{\left(\alpha_{1}, \beta_{1}\right)}(z) \\
4 \frac{(n+2) !}{n !}\left(1-z^{2}\right) P_{4}^{\left(-\alpha_{2},-\beta_{2}\right)}(z) & 0 & \frac{(3+\alpha+\beta)_{2}}{4} P_{0}^{\left(\alpha_{2}, \beta_{2}\right)}(z) & \frac{\left(1+\alpha+\beta+j_{2}\right)}{4} P_{j-2}^{\left(\alpha_{2}, \beta_{2}\right)}(z) \\
(-8) \frac{(n+3) !}{n !} P_{5}^{\left(-\alpha_{3},-\beta_{3}\right)}(z) & 0 & 0 & \frac{(1+\alpha+\beta+j)_{3}}{8} P_{j-3}^{\left(\alpha_{3}, \beta_{3}\right)}(z)
\end{array}\right| \text {, } \\
& \Pi_{-3,1,2}(z)=\left|\begin{array}{ccc}
\left(1-z^{2}\right) P_{2}^{(-\alpha,-\beta)}(z) & P_{1}^{(\alpha, \beta)}(z) & P_{2}^{(\alpha, \beta)}(z) \\
(-2)(n+1)\left(1-z^{2}\right) P_{3}^{\left(-\alpha_{1},-\beta_{1}\right)}(z) & \frac{(2+\alpha+\beta)_{1}}{2} P_{0}^{\left(\alpha_{1}, \beta_{1}\right)}(z) & \frac{(3+\alpha+\beta)_{1}}{2} P_{1}^{\left(\alpha_{1}, \beta_{1}\right)}(z) \\
4 \frac{(n+2) !}{n !} P_{4}^{\left(-\alpha_{2},-\beta_{2}\right)}(z) & 0 & \frac{(3+\alpha+\beta)_{2}}{4} P_{0}^{\left(\alpha_{2}, \beta_{2}\right)}(z)
\end{array}\right|, \\
& \Pi_{1,2,4,5}(z)=\left|\begin{array}{cccc}
P_{1}^{\left(\alpha_{-3}, \beta_{-3}\right)}(z) & P_{2}^{\left(\alpha_{-3}, \beta_{-3}\right)}(z) & P_{4}^{\left(\alpha_{-3}, \beta_{-3}\right)}(z) & P_{5}^{\left(\alpha_{-3}, \beta_{-3}\right)}(z) \\
\frac{(\alpha+\beta-4)_{1}}{2} P_{0}^{\left(\alpha_{-2}, \beta_{-2}\right)}(z) & \frac{(\alpha+\beta-3)_{1}}{2} P_{1}^{\left(\alpha_{-2}, \beta_{-2}\right)}(z) & \frac{(\alpha+\beta-1)_{1}}{2} P_{3}^{\left(\alpha_{-2}, \beta_{-2}\right)}(z) & \frac{(\alpha+\beta)_{1}}{2} P_{4}^{\left(\alpha_{-2}, \beta_{-2}\right)}(z) \\
0 & \frac{(\alpha+\beta-3)_{2}}{4} P_{0}^{\left(\alpha_{-1}, \beta_{-1}\right)}(z) & \frac{(\alpha+\beta-1)_{2}}{4} P_{2}^{\left(\alpha_{-1}, \beta_{-1}\right)}(z) & \frac{(\alpha+\beta)_{2}}{4} P_{3}^{\left(\alpha_{-1}, \beta_{-1}\right)}(z) \\
0 & 0 & \frac{(\alpha+\beta-1)_{3}}{8} P_{1}^{(\alpha, \beta)}(z) & \frac{(\alpha+\beta)_{3}}{8} P_{2}^{(\alpha, \beta)}(z) \\
0 & 0 & \frac{(\alpha+\beta-1)_{4}}{16} P_{0}^{\left(\alpha_{1}, \beta_{1}\right)}(z) & \frac{(\alpha+\beta)_{4}}{16} P_{1}^{\left(\alpha_{1}, \beta_{1}\right)}(z)
\end{array}\right|
\end{aligned}
$$

and $\Pi_{1,2,4,5, j+3}(z)$ is equal to

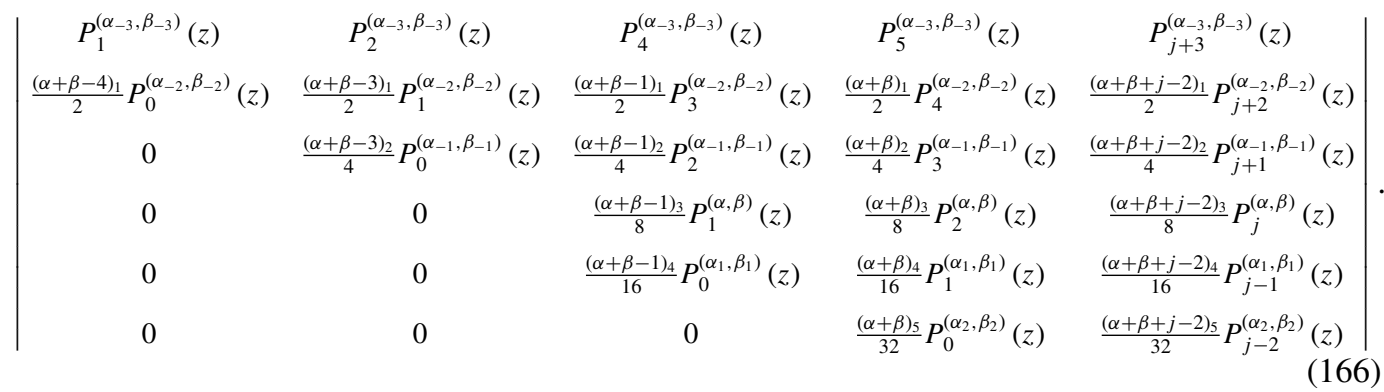

\section{THE MORSE POTENTIAL}

The Morse potential is the second exceptional primary TSIP of the first category. ${ }^{32}$ It is defined as $^{38,39}$

$$
V(y ; \omega, \alpha)=\omega^{2} y^{2}-2 \alpha \omega y+(\alpha-1 / 2)^{2}, \omega \in \mathbb{R},|\alpha|>1 / 2,
$$

where $y=\exp (-x)>0, x \in \mathbb{R}$. Contrary to the preceding examples, the Morse potential possesses only a finite number of bound states. If we choose $\omega>0, \alpha>1 / 2$, it has exactly $[\alpha-1 / 2]$ bound states ( $[a]$ being the integer part of $a$ ) which are given by

$$
\psi_{n}(x ; \omega, \alpha) \sim y^{\alpha_{n}-1 / 2} e^{-\omega y} L_{n}^{2 \alpha_{n}-1}(2 \omega y), n \in\{0, \ldots,[\alpha-3 / 2]\},
$$

with the corresponding energies $E_{n}(\alpha)=(\alpha-1 / 2)^{2}-\left(\alpha_{n}-1 / 2\right)^{2}=n(2 \alpha-1-n)$, where $\alpha_{k}$ $=\alpha-k$.

The only parameter transformation under which the Morse potential Eq. (167) is covariant, is the $\Gamma_{3}$ symmetry given by

$$
(\omega, \alpha) \stackrel{\Gamma_{3}}{\rightarrow}(-\omega,-\alpha),\left\{\begin{array}{c}
V(x ; \omega, \alpha) \stackrel{\Gamma_{3}}{\rightarrow} V(x ;-\omega,-\alpha)-E_{-1}(\alpha), \\
\psi_{n}(x ; \omega, \alpha) \stackrel{\Gamma_{3}}{\rightarrow} \psi_{n}(x ;-\omega,-\alpha) .
\end{array}\right.
$$


Applying the symmetry $\Gamma_{3}$ on the Schrödinger equation for $\psi_{n}$, we obtain

$$
\widehat{H}(\omega, \alpha) \psi_{n}(x ;-\omega,-\alpha)=\left(E_{n}(-\alpha)+E_{-1}(\alpha)\right) \psi_{n}(x ;-\omega,-\alpha)=E_{-(n+1)}(\alpha) \psi_{n}(x ;-\omega,-\alpha),
$$

where $E_{-(n+1)}(\alpha)=-(n+1)(2 \alpha+n)<0$, even if $n>[\alpha-3 / 2]$. This implies $\left((-\alpha)_{n}\right.$ $\left.=-\alpha_{-n}=-(\alpha+n)\right)$

$$
\psi_{-(n+1)}(x ; \omega, \alpha)=\psi_{n}(x ;-\omega,-\alpha)=y^{-\alpha_{-n}-1 / 2} e^{\omega y} L_{n}^{-2 \alpha_{-n}-1}(-2 \omega y), n \geq 0 .
$$

Note that if $\alpha>1 / 2$, we have $\alpha_{-n}>1 / 2$ without any restriction on $n$. Using Eq. (134), we obtain

$$
\left\{\begin{array}{c}
\psi_{-(n+1)}(x ; \omega, \alpha)_{x \rightarrow-\infty, y \rightarrow+\infty}^{\sim} y^{n-\alpha_{-n}-1 / 2} e^{\omega y} \rightarrow+\infty \\
\psi_{-(n+1)}(x ; \omega, \alpha) \underset{x \rightarrow+\infty, y \rightarrow 0^{+}}{\rightarrow}(-1)^{n} y^{-(\alpha+n+1 / 2)}(2 \alpha+2 n) \ldots(2 \alpha+n+1) \rightarrow \pm \infty,
\end{array}\right.
$$

with $\pm=(-1)^{n} \cdot \psi_{-(n+1)}$ being in the disconjugacy sector of $V(x ; \omega, \alpha)$, the disconjugacy theorem ensures that for every odd value of $n \geq 0, \psi_{-n}$ has no node on $\mathbb{R}$. Consequently, $A\left(w_{-(n+1)}\right)$ is a regular state-adding DBT and $V^{(-n)}(x ; \omega, \alpha)$ is a regular extension of $V(x ; \omega, \alpha)$. As in the case of the harmonic oscillator, there is no limitation on the possible values of $n$ since $\alpha_{-n}=\alpha+n$ $>1 / 2$ for every positive $n$. Moreover, even when $n>[\alpha-3 / 2], \psi_{-(n+1)}$ stays an adequate seed function for a state-adding DBT. This is in agreement with the fact that for every odd $n$ the one-step extension $V^{(-n)}$, entering then in the frame of the enlarged Krein-Adler theorem, has an even gap in its spectrum. The chain of complete state-adding DBT we can consider are then not limited.

From Theorem 1, we can write

$$
V^{\left(-M / / n_{1}, \ldots, n_{k}\right)}(x ; \omega, \alpha)=V^{\left(n_{1}+m, \ldots, n_{k}+m\right)}\left(x ; \omega, \alpha_{-m}\right)-(m+1)(2 \alpha+m),
$$

the eigenstates of $V^{\left(-M / / n_{1}, \ldots, n_{k}\right)}$ satisfying (see Theorem 1) for $-m \leq j \leq[\alpha-3 / 2]-m$

$$
\begin{aligned}
\psi_{j}^{\left(-M / / n_{1}, \ldots, n_{k}\right)}(x ; \omega, \alpha) & =\frac{W^{\left(-M / / n_{1}, \ldots, n_{k}, j\right)}(x ; \omega, \alpha)}{W^{\left(-M / / n_{1}, \ldots, n_{k}\right)}(x ; \omega, \alpha)} \\
& \sim \frac{W^{\left(n_{1}+m, \ldots, n_{k}+m, j+m\right)}\left(x ; \omega, \alpha_{-m}\right)}{W^{\left(n_{1}+m, \ldots, n_{k}+m\right)}\left(x ; \omega, \alpha_{-m}\right)}=\psi_{j+m}^{\left(n_{1}+m, \ldots, n_{k}+m\right)}\left(x ; \omega, \alpha_{-m}\right) .
\end{aligned}
$$

In the same example as before (see Eq. (115)),

$$
V^{(-3,1,2)}(x ; \omega, \alpha)=V^{(1,2,4,5)}(x ; \omega, \alpha)-4(2 \alpha+3)
$$

and

$$
\frac{W\left(\psi_{-3}, \psi_{1}, \psi_{2}, \psi_{j} \mid x ; \omega, \alpha\right)}{W\left(\psi_{-3}, \psi_{1}, \psi_{2} \mid x ; \omega, \alpha\right)} \sim \frac{W\left(\psi_{1}, \psi_{2}, \psi_{4}, \psi_{5}, \psi_{j+3} \mid x ; \omega, \alpha_{-3}\right)}{W\left(\psi_{1}, \psi_{2}, \psi_{4}, \psi_{5} \mid x ; \omega, \alpha_{-3}\right)}
$$

Using Eqs. (130) and (133) and usual properties of Wronskians, ${ }^{55}$ we obtain $(z=2 \omega y)$

$$
\begin{aligned}
& \frac{W\left(z^{-2 \alpha-2} e^{z} L_{2}^{-2 \alpha-3}(-z), z^{-1} L_{1}^{2 \alpha-3}(z), z^{-2} L_{2}^{2 \alpha-5}(z), z^{-j} L_{j}^{2 \alpha-2 j-1}(z) \mid x\right)}{W\left(z^{-2 \alpha-2} e^{z} L_{2}^{-2 \alpha-3}(-z), z^{-1} L_{1}^{2 \alpha-3}(z), z^{-2} L_{2}^{2 \alpha-5}(z) \mid x\right)} \\
\sim & \frac{W\left(z^{2} L_{1}^{2 \alpha+3}(z), z L_{2}^{2 \alpha+1}(z), z^{-1} L_{4}^{2 \alpha-3}(z), z^{-2} L_{5}^{2 \alpha-5}(z), z^{-j} L_{j+3}^{2 \alpha-2 j-1}(z) \mid x\right)}{W\left(z^{2} L_{1}^{2 \alpha+3}(z), z L_{2}^{2 \alpha+1}(z), z^{-1} L_{4}^{2 \alpha-3}(z), z^{-2} L_{5}^{2 \alpha-5}(z) \mid x\right)},
\end{aligned}
$$


which, with $W\left(y_{1}, \ldots, y_{m} \mid x\right)=\left(\frac{d z}{d x}\right)^{m(m-1) / 2} W\left(y_{1}, \ldots, y_{m} \mid z\right),{ }^{55}$ leads to the following bilinear Wronskian identity:

$$
\begin{aligned}
& \frac{W\left(z^{-2 \alpha-2} e^{z} L_{2}^{-2 \alpha-3}(-z), z^{-1} L_{1}^{2 \alpha-3}(z), z^{-2} L_{2}^{2 \alpha-5}(z), z^{-j} L_{j}^{2 \alpha-2 j-1}(z) \mid z\right)}{W\left(z^{-2 \alpha-2} e^{z} L_{2}^{-2 \alpha-3}(-z), z^{-1} L_{1}^{2 \alpha-3}(z), z^{-2} L_{2}^{2 \alpha-5}(z) \mid z\right)} \\
\sim & z \frac{W\left(z^{2} L_{1}^{2 \alpha+3}(z), z L_{2}^{2 \alpha+1}(z), z^{-1} L_{4}^{2 \alpha-3}(z), z^{-2} L_{5}^{2 \alpha-5}(z), z^{-j} L_{j+3}^{2 \alpha-2 j-1}(z) \mid z\right)}{W\left(z^{2} L_{1}^{2 \alpha+3}(z), z L_{2}^{2 \alpha+1}(z), z^{-1} L_{4}^{2 \alpha-3}(z), z^{-2} L_{5}^{2 \alpha-5}(z) \mid z\right)} .
\end{aligned}
$$

\section{THE EFFECTIVE RADIAL KEPLER-COULOMB (ERKC)}

The ERKC potential with zero ground level $\left(E_{0}(\alpha)=0\right)$ is the third and last exceptional primary TSIP of the first category. ${ }^{32}$ It is defined on the positive half line $x>0$ as

$$
V(x ; \alpha)=\frac{(\alpha+1 / 2)(\alpha-1 / 2)}{x^{2}}-\frac{\gamma}{x}+\frac{\gamma^{2}}{4(\alpha+1 / 2)^{2}}, \gamma>0,|\alpha|>1 / 2
$$

Choosing $\alpha>1 / 2$, its bound states are given by $\left(z_{n}=\gamma x /\left(\alpha_{n}+1 / 2\right)\right)$

$$
\psi_{n}(x ; \alpha)=x^{\alpha+1 / 2} e^{-z_{n} / 2} L_{n}^{2 \alpha}\left(z_{n}\right), n \geq 0,
$$

with the corresponding energies

$$
E_{n}(\alpha)=\frac{\gamma^{2}}{4(\alpha+1 / 2)^{2}}-\frac{\gamma^{2}}{4\left(\alpha_{n}+1 / 2\right)^{2}},
$$

where $\alpha_{k}=\alpha+k$.

The only covariance transformation for the ERKC potentials is the $\Gamma_{3}$ is given by

$$
\alpha \stackrel{\Gamma_{3}}{\rightarrow}(-\alpha),\left\{\begin{array}{c}
V(x ; \alpha) \stackrel{\Gamma_{3}}{\rightarrow} V(x ; \alpha)-E_{-1}(\alpha), \\
\psi_{n}(x ; \alpha) \stackrel{\Gamma_{3}}{\rightarrow} \psi_{n}(x ;-\alpha),
\end{array}\right.
$$

with

$$
\begin{aligned}
\alpha_{k} \stackrel{\Gamma_{3}}{\rightarrow}-\alpha+k & =-\alpha_{-k}, \quad E_{n}(\alpha) \stackrel{\Gamma_{3}}{\rightarrow} \gamma^{2} / 4\left(\frac{1}{\left(\alpha_{-1}+1 / 2\right)^{2}}-\frac{1}{\left(\alpha_{-(n+1)}+1 / 2\right)^{2}}\right) \\
& =E_{-(n+1)}(\alpha)-E_{-1}(\alpha) .
\end{aligned}
$$

We then have

$$
\widehat{H}(x ; \alpha) \psi_{n}(x ;-\alpha)=E_{-(n+1)}(\alpha) \psi_{n}(x ;-\alpha),
$$

which leads to

$$
\psi_{-(n+1)}(x ; \alpha)=\psi_{n}(x ;-\alpha)=x^{-\alpha+1 / 2} \exp \left(z_{-(n+1)} / 2\right) L_{n}^{-2 \alpha}\left(-z_{-(n+1)}\right) .
$$

Since we have $E_{-(n+1)}(\alpha)=1 /(\alpha+1 / 2)^{2}-1 /\left(\alpha_{-(n+1)}+1 / 2\right)^{2}<0$ for every value of $n$ $\geq 0, \psi_{-(n+1)}(x ; \alpha)$ is in the disconjugacy sector of $V(x ; \alpha)$. Using Eq. (134) and the disconjugacy theorem, we deduce that, if $\alpha>n+1 / 2$

$$
\left\{\begin{array}{c}
\psi_{-(n+1)}(x ; \alpha) \underset{x \rightarrow 0^{+}}{\sim} x^{-\alpha+1 / 2 \frac{(-2)^{n}}{n !}(\alpha-1 / 2) \ldots(\alpha-n) \rightarrow \pm \infty} \\
\psi_{-(n+1)}(x ; \alpha) \underset{x \rightarrow+\infty}{\sim} \frac{x^{n-\alpha+1 / 2}}{(\alpha-n-1 / 2)^{n} n !} \exp \left(\frac{\gamma x}{2(\alpha-n-1 / 2)}\right) \rightarrow+\infty
\end{array}\right.
$$


with $\pm=(-1)^{n}$. Consequently, $\psi_{-n}$ is free of node for node for $n$ odd and $A\left(w_{-n}\right)$ is then a state-adding DBT. If $1 / 2<\alpha<n+1 / 2$

$$
\left\{\begin{array}{c}
\psi_{-(n+1)}(x ; \alpha) \underset{x \rightarrow 0^{+}}{\sim} x^{-\alpha+1 / 2} \frac{2^{n}}{n !}(\alpha-1 / 2) \ldots(\alpha-n) \rightarrow \pm \infty, \\
\psi_{-(n+1)}(x ; \alpha) \underset{x \rightarrow+\infty}{\sim} \frac{x^{n-\alpha+1 / 2}}{(\alpha-n-1 / 2)^{n} n !} \exp \left(\frac{\gamma x}{2(\alpha-n-1 / 2)}\right) \rightarrow 0^{ \pm},
\end{array}\right.
$$

and although $A\left(w_{-n}\right)$ is regular, it is not state-adding and $V^{(-n)}$ is strictly isospectral to $V$, as already noted in Ref. 32 by using the Kienast-Lawton-Hahn theorem. Note that the constraint is coherent with the fact that the application of the enlarged Krein-Adler theorem requires, as in the case of the isotonic oscillator, the assumption of the regularity of all the extensions $V^{(-j)}$ of the complete chain $(-M)$, namely, the condition $\left|\alpha_{-j}\right|<1 / 2, \forall j \leq m$.

If $\alpha>m+1 / 2$, the extended potential $V^{\left(-M / / n_{1}, \ldots, n_{k}\right)}$ is regular and satisfies (see Theorem 1)

$$
V^{\left(-M / / n_{1}, \ldots, n_{k}\right)}(x ; \alpha)=V^{\left(n_{1}+m, \ldots, n_{k}+m\right)}\left(x ; \alpha_{-m}\right)-2 m \omega,
$$

with eigenstates $(j \geq-m)$

$$
\begin{aligned}
\psi_{j}^{\left(-M / / n_{1}, \ldots, n_{k}\right)}(x ; \alpha) & =\frac{W^{\left(-M / / n_{1}, \ldots, n_{k}, j\right)}(x ; \alpha)}{W^{\left(-M / / n_{1}, \ldots, n_{k}\right)}(x ; \alpha)} \sim \frac{W^{\left(n_{1}+m, \ldots, n_{k}+m, j+m\right)}\left(x ; \alpha_{-m}\right)}{W^{\left(n_{1}+m, \ldots, n_{k}+m\right)}\left(x ; \alpha_{-m}\right)} \\
& =\psi_{j+m}^{\left(n_{1}+m, \ldots, n_{k}+m\right)}\left(x ; \alpha_{-m}\right) .
\end{aligned}
$$

For instance, (see Eq. (115)) for $\alpha>7 / 2$

$$
V^{(-3,1,2)}(x ; \alpha)=V^{(1,2,4,5)}(x ; \alpha)-3 \omega
$$

and

$$
\frac{W\left(\psi_{-3}, \psi_{1}, \psi_{2}, \psi_{j} \mid x ; \omega, \alpha\right)}{W\left(\psi_{-3}, \psi_{1}, \psi_{2} \mid x ; \omega, \alpha\right)} \sim \frac{W\left(\psi_{1}, \psi_{2}, \psi_{4}, \psi_{5}, \psi_{j+3} \mid x ; \omega, \alpha_{-3}\right)}{W\left(\psi_{1}, \psi_{2}, \psi_{4}, \psi_{5} \mid x ; \omega, \alpha_{-3}\right)} .
$$

Using Eqs. (180) and (185) and usual properties of Wronskians, ${ }^{55}$ this leads to the following bilinear Wronskian identity:

$$
\begin{aligned}
& \frac{W\left(x^{-2 \alpha} e^{z_{-3} / 2} L_{2}^{-2 \alpha}\left(-z_{-3}\right), e^{-z_{1} / 2} L_{1}^{2 \alpha}\left(z_{1}\right), e^{-z_{2} / 2} L_{2}^{2 \alpha}(z), e^{-z_{j} / 2} L_{j}^{2 \alpha}(z) \mid x\right)}{W\left(x^{-2 \alpha} e^{z_{-2} / 2} L_{2}^{-2 \alpha}\left(-z_{-2}\right), e^{-z_{1} / 2} L_{1}^{2 \alpha}\left(z_{1}\right), e^{-z_{2} / 2} L_{2}^{2 \alpha}(z) \mid x\right)} \\
\sim & \frac{W\left(e^{-z_{-2} / 2} L_{1}^{\alpha_{-3}}\left(z_{-2}\right), e^{-z_{-1} / 2} L_{2}^{\alpha_{-3}}\left(z_{-1}\right), e^{-z_{1} / 2} L_{4}^{\alpha_{-3}}\left(z_{1}\right), e^{-z_{2} / 2} L_{5}^{\alpha_{-3}}\left(z_{2}\right), e^{-z_{j} / 2} L_{j+3}^{\alpha_{-3}}\left(z_{j}\right) \mid x\right)}{W\left(e^{-z_{-2} / 2} L_{1}^{\alpha_{-3}}\left(z_{-2}\right), e^{-z_{-1} / 2} L_{2}^{\alpha_{-3}}\left(z_{-1}\right), e^{-z_{1} / 2} L_{4}^{\alpha_{-3}}\left(z_{1}\right), e^{-z_{2} / 2} L_{5}^{\alpha_{-3}}\left(z_{2}\right) \mid x\right)} .
\end{aligned}
$$

\section{CONCLUSION}

We have shown that, when applied to PTSIP, the Krein-Adler theorem admits a generalization which gives the regularity condition for mixed chains of state-deleting and state-adding DBT, these lasts being associated to type 3 seed functions. Moreover, the existence of a reverse shape invariance condition for complete chains of state-adding DBT leads to new bilinear determinantal identities for the classical orthogonal polynomials.

A more general version of this enlarged Krein-Adler theorem applicable to a wider class of potentials will be addressed in a forthcoming work.

For the specific case of PTSIP, the question of the regularity condition for chains mixing all the different types of quasi-polynomials seed functions (associated to state-adding, state-deleting, and strictly isospectral DBT) is the object of further investigations.

${ }^{1}$ G. Darboux, "Sur une proposition relative aux équations linéaires," C. R. Acad. Sci. 94, 1456-1459 (1882); http://arxiv.org/pdf/physics/9908003.pdf.

${ }^{2}$ M. M. Crum, "Associated Sturm-Liouville systems," Q. J. Math. 6, 121-127 (1955).

${ }^{3}$ E. Schrödinger, "A method of determining quantum-mechanical eigenvalues and eigenfunctions," Proc. R. Irish Acad. A46, 9-16 (1940); http://www.jstor.org/stable/20490744. 
${ }^{4}$ L. Infeld and T. E. Hull, “The factorization method,” Rev. Mod. Phys. 23, 21-68 (1951).

${ }^{5}$ D. Gómez-Ullate, N. Kamran, and R. Milson, "The Darboux transformation and algebraic deformations of shape invariant potentials," J. Phys. A 37, 1789-1804 (2004).

${ }^{6}$ D. Gómez-Ullate, N. Kamran, and R. Milson, "Supersymmetry and algebraic Darboux transformations," J. Phys. A 37, $10065-10078$ (2004).

${ }^{7}$ D. Gómez-Ullate, N. Kamran, and R. Milson, "An extended class of orthogonal polynomials defined by a Sturm-Liouville problem,” J. Math. Anal. Appl. 359, 352 (2009).

${ }^{8}$ D. Gómez-Ullate, N. Kamran, and R. Milson, "An extension of Bochner's problem: Exceptional invariant subspaces," J. Approx. Theory 162, 987-1006 (2010).

${ }^{9}$ D. Gómez-Ullate, N. Kamran, and R. Milson, "Exceptional orthogonal polynomials and the Darboux transformation," J. Phys. A 43, 434016 (2010).

${ }^{10}$ D. Gómez-Ullate, N. Kamran, and R. Milson, "On orthogonal polynomials spanning a non-standard flag," in Algebraic Aspects of Darboux Transformations, Quantum Integrable Systems and Supersymmetric Quantum Mechanics, Contemporary Mathematics Vol. 563, edited by P. Acosta-Humanez, F. Finkel, N. Kamran, and P. Olver (AMS, 2012).

${ }^{11}$ D. Gómez-Ullate, N. Kamran, and R. Milson, "Two-step Darboux transformations and exceptional Laguerre polynomials," J. Math. Anal. Appl. 387, 410-418 (2012).

${ }^{12}$ D. Gómez-Ullate, N. Kamran, and R. Milson, "A conjecture on exceptional orthogonal polynomials," Found. Comput. Math. 13, 615-666 (2013).

${ }^{13}$ C. Quesne, "Exceptional orthogonal polynomials, exactly solvable potentials and supersymmetry,” J. Phys. A 41, 392001 (2008).

${ }^{14}$ C. Quesne, "Solvable rational potentials and exceptional orthogonal polynomials in supersymmetric quantum mechanics," SIGMA 5, 084 (2009).

${ }^{15}$ B. Bagchi, C. Quesne, and R. Roychoudhury, "Isospectrality of conventional and new extended potentials, second-order supersymmetry and role of $\mathcal{P} \mathcal{T}$ symmetry," Pramana J. Phys. 73, 337-347 (2009).

${ }^{16}$ B. Bagchi and C. Quesne, "An update on $\mathcal{P} \mathcal{T}$-symmetric complexified Scarf II potential, spectral singularities and some remarks on the rationally-extended supersymmetric partners," J. Phys. A 43, 305301 (2010).

${ }^{17} \mathrm{~S}$. Odake and R. Sasaki, "Infinitely many shape invariant potentials and new orthogonal polynomials," Phys. Lett. B 679, 414-417 (2009).

${ }^{18}$ S. Odake and R. Sasaki, "Another set of infinitely many exceptional $\left(\mathrm{X}_{l}\right)$ Laguerre polynomials," Phys. Lett. B 684, $173-176(2010)$.

${ }^{19}$ C.-L. Ho, S. Odake, and R. Sasaki, "Properties of the exceptional $\left(\mathrm{X}_{l}\right)$ Laguerre and Jacobi polynomials," SIGMA 7, 107 (2011).

${ }^{20}$ S. Odake and R. Sasaki, "Infinitely many shape invariant potentials and cubic identities of the Laguerre and Jacobi polynomials,” J. Math. Phys. 51, 053513 (2010).

${ }^{21}$ R. Sasaki, S. Tsujimoto, and A. Zhedanov, "Exceptional Laguerre and Jacobi polynomials and the corresponding potentials through Darboux-Crum transformations," J. Phys. A 43, 315204 (2010).

${ }^{22}$ S. Odake and R. Sasaki, "Exactly solvable quantum mechanics and infinite families of multi-indexed orthogonal polynomials," Phys. Lett. B 702, 164-170 (2011).

${ }^{23}$ S. Odake and R. Sasaki, "Krein-Adler transformations for shape-invariant potentials and pseudo virtual states," J. Phys. A 46, 245201 (2013).

${ }^{24}$ S. Odake and R. Sasaki, "Extensions of solvable potentials with finitely many discrete eigenstates," J. Phys. A 46, 235205 (2013).

${ }^{25}$ D. Dutta and P. Roy, “Conditionally exactly solvable potentials and exceptional orthogonal polynomials,” J. Math. Phys. 51, 042101 (2010).

${ }^{26}$ C.-L. Ho and R. Sasaki, "Zeros of the exceptional Laguerre and Jacobi polynomials," SIGMA 7, 107 (2011).

${ }^{27}$ C.-L. Ho, R. Sasaki, and K. Takemura, "Confluence of apparent singularities in multi-indexed orthogonal polynomials: The Jacobi case,” J. Phys. A 46, 115205 (2013).

${ }^{28}$ Y. Grandati and A. Bérard, "Solvable rational extension of translationally shape invariant potentials," in Algebraic Aspects of Darboux Transformations, Quantum Integrable Systems and Supersymmetric Quantum Mechanics, Contemporary Mathematics Vol. 563, edited by P. Acosta-Humanez, F. Finkel, N. Kamran, and P. Olver (AMS, 2012).

${ }^{29}$ Y. Grandati, "Solvable rational extensions of the isotonic oscillator," Ann. Phys. 326, 2074-2090 (2011).

${ }^{30}$ Y. Grandati, "Solvable rational extensions of the Morse and Kepler-Coulomb potentials," J. Math. Phys. 52, 103505 (2011).

${ }^{31}$ Y. Grandati, "Multistep DBT and regular rational extensions of the isotonic oscillator," Ann. Phys. 327, 2411 (2012).

${ }^{32}$ Y. Grandati and A. Bérard, "Rational solutions for the Riccati-Schrödinger equations associated to translationally shape invariant potentials," Ann. Phys. 325, 1235-1259 (2010).

${ }^{33}$ Y. Grandati, "New rational extensions of solvable potentials with finite bound state spectrum," Phys. Lett. A 376, 2866-2872 (2012).

${ }^{34}$ Y. Grandati and C. Quesne, "Disconjugacy, regularity of multi-indexed rationally-extended potentials, and Laguerre exceptional polynomials," J. Math. Phys. 54, 073512 (2013).

${ }^{35}$ Y. Grandati and A. Bérard, "Comments on the generalized SUSY QM partnership for Darboux-Pöschl-Teller potential and exceptional Jacobi polynomials," J. Eng. Math. 82, 161-171 (2013).

${ }^{36}$ A. Ramos, "On the new translational shape invariant potentials," J. Phys. A 44, 342001 (2011).

${ }^{37}$ A. Ramos, "Symmetries and the compatibility condition for the new translational shape invariant potentials," Phys. Lett. A 376, 3499-3503 (2012).

${ }^{38}$ F. Cooper, A. Khare, and U. Sukhatme, Supersymmetry in Quantum Mechanics (World Scientific, Singapore, 2001). 
${ }^{39}$ R. Dutt, A. Khare, and U. P. Sukhatme, "Supersymmetry, shape invariance and exactly solvable potentials," Am. J. Phys. 56, 163-168 (1988).

${ }^{40}$ L. Gendenshtein, "Derivation of exact spectra of the Schrodinger equation by means of supersymmetry," JETP Lett. 38, 356-359 (1983); http://www.jetpletters.ac.ru/ps/1822/article_27857.shtml.

${ }^{41}$ C. V. Sukumar, "Supersymmetric quantum mechanics of one-dimensional systems," J. Phys. A 18, 2917-2936 (1985).

${ }^{42}$ D. Gómez-Ullate, Y. Grandati, and R. Milson, "Rational extensions of the quantum harmonic oscillator and exceptional Hermite polynomials," J. Phys. A 47, 015203 (2014).

${ }^{43}$ P. Hartman, Ordinary Differential Equations (John Wiley, New York, 1964).

${ }^{44}$ W. A. Coppel, Disconjugacy (Springer, Berlin, 1971).

${ }^{45}$ M. Bôcher, Leçons sur les méthodes de Sturm (Gauthier-Villars, Paris, 1917), pp. 51-52.

${ }^{46}$ V. Y. Derr, "The theory of disconjugacy for a second order linear differential equations," e-print arXiv:0811.4636 [mathCA].

${ }^{47}$ G. Szegö, Orthogonal Polynomials (American Mathematical Society, Providence, 1975).

${ }^{48}$ A. Erdélyi, W. Magnus, F. Oberhettinger, and F. G. Tricomi, Higher Transcendental Functions (McGraw-Hill, New York, 1953).

${ }^{49}$ C. Quesne, "Higher-order SUSY, exactly solvable potentials, and exceptional orthogonal polynomials," Mod. Phys. Lett. A 26, 1843-1852 (2011).

${ }^{50} \mathrm{C}$. Quesne, "Rationally-extended radial oscillators and Laguerre exceptional orthogonal polynomials in kth-order SUSYQM,” Int. J. Mod. Phys. A 26, 5337-5347 (2011).

${ }^{51} \mathrm{C}$. Quesne, "Novel enlarged shape invariance property and exactly solvable rational extensions of the Rosen-Morse II and Eckart potentials," SIGMA 8, 080 (2012).

${ }^{52}$ C. Quesne, "Revisiting (quasi-)exactly solvable rational extensions of the Morse potential," Int. J. Mod. Phys. A 27, 1250073 (2012).

${ }^{53}$ J. F. Cariñena and A. Ramos, "Integrability of Riccati equation from a group theoretical viewpoint," Int. J. Mod. Phys. A 14, 1935-1951 (1999).

${ }^{54}$ J. F. Cariñena, A. Ramos, and D. J. Fernandez, "Group theoretical approach to the intertwined hamiltonians," Ann. Phys. 292, 42-66 (2001)

${ }^{55}$ T. Muir, A Treatise on the Theory of Determinants (Dover, New York, 1960) (revised and enlarged by W. H. Metzler).

${ }^{56}$ R. Vein and P. Dale, Determinants and their Applications in Mathematical Physics (Springer-Verlag, New York, 1999).

${ }^{57}$ M. G. Krein, "On a continuous analogue of a Christoffel formula from the theory of orthogonal polynomials," Dokl. Akad. Nauk SSSR 113, 970 (1957).

${ }^{58}$ V. E. Adler, "A modification of Crum method," Theor. Math. Phys. 101, 1381-1386 (1994)

${ }^{59}$ V. B. Matveev and M. A. Salle, Darboux Transformations and Solitons (Springer-Verlag, Berlin, 1991).

${ }^{60}$ C. V. Sukumar, "Supersymmetry, factorization of the Schrödinger equation and an hamiltonian hierarchy," J. Phys. A 18, L57-L61 (1985)

${ }^{61}$ A. A. Andrianov, N. V. Borisov, and M. V. Ioffe, "The factorization method and quantum systems with equivalent energy spectra," Phys. Lett. A 105, 19-22 (1984).

${ }^{62}$ A. A. Andrianov, M. V. Ioffe, and V. P. Spiridonov, "Higher-derivative supersymmetry and the Witten index," Phys. Lett. A 174, 273-279 (1993).

${ }^{63}$ V. G. Bagrov and B. F. Samsonov, "Darboux transformation, factorization, and supersymmetry in one-dimensional quantum mechanics," Theor. Math. Phys. 104, 1051-1060 (1995).

${ }^{64}$ V. G. Bagrov and B. F. Samsonov, "Darboux transformation and elementary exact solutions of the Schrödinger equation," Pramana J. Phys. 49, 563-580 (1997).

${ }^{65}$ B. F. Samsonov and I. N. Ovcharov, "Darboux transformation and exactly solvable potentials with quasi-equidistant spectrum,” Russ. Phys. J. 38, 765-771 (1995).

${ }^{66}$ V. G. Bagrov, B. F. Samsonov, and L. A. Shekoyan, "N-order Darboux transformation and a spectral problem on semiaxis," e-print arXiv:9804.032

${ }^{67}$ B. F. Samsonov, "New possibilities for supersymmetry breakdown in quantum mechanics and second order irreducible Darboux transformations," Phys. Lett. A 263, 274-280 (1999).

${ }^{68}$ D. J. Fernández C, V. Hussin, and B. Mielnik, "A simple generation of exactly solvable anharmonic oscillators," Phys. Lett. A 244, 309-316 (1998).

${ }^{69}$ D. J. Fernández C, J. Negro, and L. M. Nieto, "Second-order supersymmetric periodic potentials," Phys. Lett. A 275, 338-349 (2000).

${ }^{70}$ D. J. Fernández C, R. Muñoz, and A. Ramos, "Second order SUSY transformations with "complex energies",” Phys. Lett. A 308, 11-16 (2003).

${ }^{71}$ B. Mielnik, L. M. Nieto, and O. Rosas-Ortiz, "The finite difference algorithm for higher order supersymmetry," Phys. Lett. A 269, 70-78 (2000).

${ }^{72}$ A. A. Oblomkov, "Monodromy-free Schrödinger operators with quadratically increasing potentials," Theor. Math. Phys. 121, 1574-1584 (1999).

${ }^{73}$ J. J. Duistermaat and F. A. Grünbaum, "Differential equations in the spectral parameter," Commun. Math. Phys. 103, 177-240 (1986).

${ }^{74}$ O. A. Chalykh, "Darboux transformations for multidimensional Schrödinger operators," Russ. Math. Surv. 53, 377-379 (1998).

${ }^{75}$ Y. Y. Berest and A. P. Veselov, "On the singularities of potentials of exactly soluble Schrödinger equations and on Hadamard's problem,” Russ. Math. Surv. 53, 208-209 (1998). 
${ }^{76}$ V. M. Goncharenko and A. P. Veselov, "Monodromy of the matrix Schrödinger equations and Darboux transformations," J. Phys. A 31, 5315-5326 (1998).

${ }^{77}$ E. E. Shnol', Appendix B in S. Dubov, V. M. Eleonskii, and N. E. Kulagin, "Equidistant spectra of anharmonic oscillators," Chaos 4, 47-53 (1994).

${ }^{78}$ V. M. Tkachuk, "Supersymmetric method for constructing quasi-exactly and conditionally-exactly solvable potentials," J. Phys. A 32, 1291-1312 (1999).

${ }^{79}$ G. E. Andrews, R. Askey, and R. Roy, Special Functions (Cambridge University Press, Cambridge, 2000).

${ }^{80}$ A. Messiah, Mécanique Quantique T1 (Dunod, Paris, 1969). 